\title{
Structural and magnetic disorder in crystalline materials: a first principles study
}

Davide Gambino

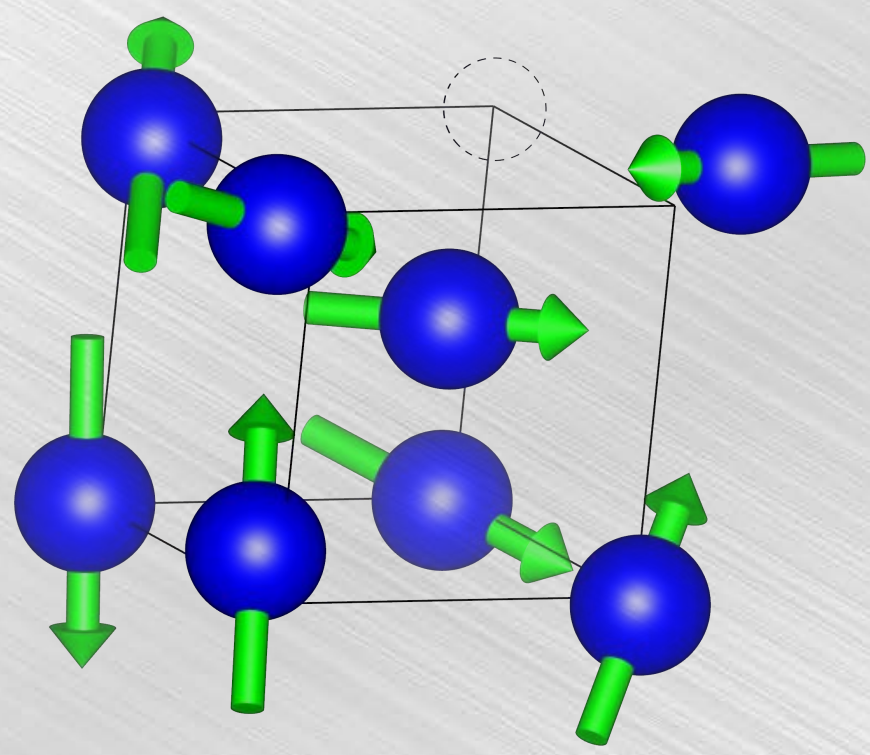


Linköping Studies in Science and Technology

Thesis No. 1837

\title{
Structural and magnetic disorder in crystalline materials: a first principle study
}

\author{
Davide Gambino
}


The front page illustrates a snapshot of an atomistic spin dynamics-ab initio molecular dynamics simulation of bcc Fe with a vacancy. The conventional cubic cell is shown with black lines, with atoms vibrating around equilibrium positions. The green arrows represent the local magnetic moments.

During the course of research underlying this thesis, Davide Gambino was enrolled in Agora Materiae, a multidiciplinary doctoral program at Linköping University, Sweden.

(C) Davide Gambino

ISBN 978-91-7685-081-7

ISSN 0280-7971

Printed by LiU-Tryck 2019 
"Nel mezzo del cammin di nostra vita..." The Divine Comedy, Dante Alighieri, 1320 A.D. 



\section{Abstract}

Disorder in crystalline materials can take different forms and originate from different sources. In particular, temperature introduces disorder in any kind of material. This can be observed as the appearance of vacant lattice sites in an otherwise perfect crystal, or as a random distribution of different elements on the same lattice in an alloy; at the same time, if the material is magnetic, temperature induces disorder also on the magnetic degrees of freedom. In this thesis, different levels of disorder associated to structure and magnetism are investigated by means of density functional theory and thermodynamic models.

I start with diffusion of $\mathrm{Ti}$ vacancies in $\mathrm{TiN}$, which is studied by means of nonequilibrium $a b$ initio molecular dynamics using the color diffusion algorithm at different temperatures. The result is an Arrhenius behavior of Ti vacancy jump rates.

A method to perform structural relaxations in magnetic materials in their hightemperature paramagnetic phase is then developed based on the disordered local moments approach in order to study vacancies, interstitial atoms, and combinations of defects in paramagnetic bcc Fe and $\mathrm{B} 1 \mathrm{CrN}$, as well as the mixing enthalpy of bcc $\mathrm{Fe}_{1-x} \mathrm{Cr}_{x}$ random alloys. A correction to the energetics of every system due to the relaxation in the disordered magnetic state is observed in all cases.

Not related to temperature and disorder, but very important for an accurate description of magnetic materials, is the choice of the exchange and correlation functional to be employed in the first principles calculations. We have investigated the performance of a recently developed meta-GGA functional, the strongly constrained and appropriately normed (SCAN) functional, in comparison with the more commonly used LDA and PBE on the ferromagnetic elemental solids bcc Fe, fcc $\mathrm{Ni}$, and hcp Co, and SCAN it is found to give negligible improvements, if not a worsening, in the description of these materials.

Finally, the coupling between vibrational and magnetic degrees of freedom is discussed by reviewing the literature and proposing an investigation of the influ- 
ence of vibrations on longitudinal spin fluctuations. These excitations are here studied by means of thermodynamic models based on Landau expansion of the energy in even powers of the magnitude of the local magnetic moments. We find that vibrational and magnetic disorder alter the energy landscapes as a function of moment size also in bcc Fe, which is often considered a Heisenberg system, inducing a more itinerant electron behavior. 


\section{Acknowledgements}

This thesis is just an intermediate achievement on the long journey towards the PhD degree; nonetheless, many people need to be acknowledged for their help and their patience that enabled me to get, at least, to this point.

Above all, I thank with the most gratitude my supervisor Dr. Björn Alling for teaching me how to think in physics terms, being always present, and leading my scientific growth. I still have a lot to learn, but with your guidance I am sure it is going to work out. Thanks as well for the frequent updates on Swedish politics.

A great thank goes to Prof. Igor Abrikosov for the chance he gave me to stay at Linköping University, and suggesting me to contact Björn for the $\mathrm{PhD}$ position I am currently occupying.

I thank particularly Dr. Davide Sangiovanni for the many discussions and updates on ongoing projects, which acquire a very pleasant taste when told in Italian.

A big thank as well to Dr. Irina Stockem for her support with the ASD-AIMD code and patience with my annoying and recurrent requests; also, thank you very much for hosting me at MPIE during my visits.

I must acknowledge Johan Klarbring for useful discussions and suggestions on project-related topics, and together with Johan Jönsson for bearing me at the APS 2019 March Meeting. Thanks to Johan Tidholm for the good time in the shared office (and the "Friday after $3 \mathrm{pm}$ " ritual), Dr. Marcus Ekholm for suggestion of the SCAN project, and all the other members of the Theoretical Physics group here at Linköping University.

Many thanks for interesting discussions to our collaborators at the Max-PlanckInstitute für Eisenforschung in Düsseldorf, in particular Dr. Tilmann Hickel, Dr. Blazej Grabowski, Dr. Fritz Körmann, and Dr. Liam Huber.

A special thank to Prof. Andrei Ruban for sharing his great knowledge with me, and explaining me simple things that I do not understand. Dr. Oleg Peil is acknowledge as well for the time he dedicated to me during my visit to the 
Materials Center Leoben.

Life is not made of only work, I therefore need to acknowledge all my friends here in Linköping that, through lunches, evenings and various activities, have helped me to distract and regain energies: Hassan Abdalla, Anna-Giulia Scaglia, Arnaud Le Febvrier, Johan Nyman, Max Karlsson, Clio Azina, Claudia Schnitter, Laurent Souqui, Tim Cornelissen, Andreas Jamnig, Marius Rodner, and many others.

A special mention is owed to my dear flatmates Victor Gervilla and Marianne Kropf, who created a perfect home environment and have dealt with me in stressful periods. Thanks a lot!

My last thought is for my family: my brother Gianluca and his wife Marta, who are always so caring, and the newcomer Gioeliño; my parents who have always supported me and enabled me to embark on this path. Grazie di tutto! 
1 Introduction $\quad 1$

1.1 Structural disorder . . . . . . . . . . . . . . . . . . 2

1.1.1 Defects in materials . . . . . . . . . . . . 2

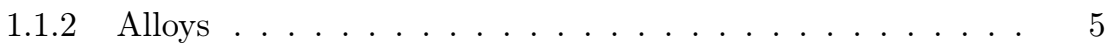

1.2 Magnetism in materials . . . . . . . . . . . . . . . . 6

1.2.1 Low temperature ordered structures . . . . . . . . . . 7

1.2.2 Thermal excitations and the paramagnetic phase . . . . . 7

1.2.3 Itinerant vs localized moments models . . . . . . . . . . 8

2 Theoretical methods 9

2.1 Density functional theory . . . . . . . . . . . . . . . . . 9

2.1.1 The Schrödinger equation . . . . . . . . . . . . 10

2.1.2 The Hohenberg-Kohn theorems and the Kohn-Sham ansatz 11

2.1.3 Exchange and correlation functional . . . . . . . . . . 13

2.1.4 Spin-polarized DFT . . . . . . . . . . . . . . 14

2.2 Thermodynamic simulations . . . . . . . . . . . . . . 15

2.2 .1 Free energy . . . . . . . . . . . . . . 16

2.2.2 Ab initio molecular dynamics . . . . . . . . . . . . 18

2.2.3 Nonequilibrium molecular dynamics - Color Diffusion algorithm .......................... 19

2.3 Ab initio description of structural disorder . . . . . . . . . . . . 22

2.3.1 Point defects . . . . . . . . . . . . . . 23

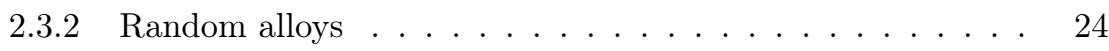

2.4 Microscopic description of magnetism . . . . . . . . . . . . . . 25

2.4.1 Heisenberg Hamiltonian . . . . . . . . . . . . . . . . 26

2.4.2 Longitudinal spin fluctuations . . . . . . . . . . . . . . . . 27

2.4.3 Disordered local moment approach . . . . . . . . . . . 30 
2.4.4 Coupled atomistic spin dynamics - ab initio molecular dynamics ...................... 32

3 Results $\quad 35$

3.1 Ti vacancy diffusion in $\mathrm{TiN}$. . . . . . . . . . . . . . . . 35

3.2 Lattice relaxation in magnetic materials in the high temperature paramagnetic phase . . . . . . . . . . . . . 38

3.2.1 Fe-based materials . . . . . . . . . . . . . . . . . . . . . 38

3.2.2 Defects in paramagnetic $\mathrm{B} 1 \mathrm{CrN}$. . . . . . . . . . 41

3.3 Assessing the SCAN functional for ferromagnetic elemental solids . 45

3.4 Interplay between vibrational, transverse, and longitudinal magnetic degrees of freedom in bcc $\mathrm{Fe}$. . . . . . . . . . . . . 46

4 Conclusions and outlooks $\quad 53$

$\begin{array}{ll}\text { Bibliography } & 55\end{array}$

$\begin{array}{ll}\text { List of included publications } & 65\end{array}$

$\begin{array}{ll}\text { Not included publications } & 67\end{array}$

5 Summary of included publications $\quad 69$

$\begin{array}{ll}\text { Paper I } & 71\end{array}$

$\begin{array}{lr}\text { Paper II } & 87\end{array}$

$\begin{array}{lr}\text { Paper III } & 99\end{array}$ 


\section{CHAPTER 1}

Introduction

The study of materials properties is a very fascinating discipline. Mankind has always tried to produce better and better materials for a wide variety of applications, and in order to do this one needs to understand where the exceptional properties of a particular system stem from, to be able to improve them even further. Since the discovery of the atomic nature of matter and the development of theories like Quantum Mechanics and Statistical Mechanics, our understanding has greatly increased and we have reached now the ability to theoretically predict properties before observing them in experiments (see Nobel prize for Physics 2016 to Thouless, Haldane, and Kosterlitz "for theoretical discoveries of topological phase transitions and topological phases of matter").

In principle, to simulate from first principles one mole (i.e., a few grams) of a real material, one should solve the Schrödinger equation for $\sim 6 \times 10^{23}$ atoms or molecules and all the electrons contained in the system, which can vary from one electron per atom in hydrogen, up to $\sim 90$ for the heaviest natural elements. This is an unattainable aim, so we need to employ approximations. At high temperatures, the quantum nature of nuclei in solids can be often disregarded, so that they can be described as classical particles acting in a potential created by the electrons and move according to Newton's equations of motion, reducing the complexity of the problem. For the electrons, though, one still has to solve the Schrödinger equation, and an efficient reformulation of this problem is the density functional theory (DFT) [1, 2], although also at this level approximations need to be made.

One of the main focuses in current theoretical materials science is the inclusion of temperature effects in simulations. These have been proven to be critical for many systems [3-5], and in this work I will try to describe how thermal fluctuations introduce a certain degree of disorder in two different areas: the first one is structural disorder, concerning the presence of vacant sites or other types of defects in crystalline materials, and randomness in the position of different chemical 
species in alloys at high temperature; the second one is magnetic disorder, related to the loss of long-range order of atomic magnetic moments in magnetic materials above the critical temperature. Both types of disorder affect the properties of materials, as will be shown throughout this thesis, so that it is very important to investigate these phenomena and obtain a deep understanding in order to model reality as accurately as possible.

\subsection{Structural disorder}

Matter in solid state can be mainly found in two forms: crystalline or amorphous. The latter, which is not the focus of this thesis, is a nonequilibrium state that can be seen as a distribution of atoms with short- but no long-range order, atomistically more similar to a liquid than a solid. The former, instead, consists of an ordered arrangement of atoms that shows at least a periodic structure with translational symmetry. If we neglect quantum effects such as the zero-point motion, at zero temperature the most energetically favored state for a crystal is with all the atoms exactly in their equilibrium lattice positions.

At low temperatures, atoms vibrate around their equilibrium positions, so that harmonic phonons describe well this regime; however, this means as well that a classical description of the system is not accurate enough, since phonons arise from the quantum nature of atoms. Above the Debye temperature of the system, the classical picture can be used but, for temperatures close to melting, vibrations become so large that the harmonic approximation does not hold anymore and anharmonic effects become important.

Other sources of disorder in crystals are point defects and configurational disorder in alloys, which are intimately connected to the thermodynamics of the system. Examples of different kinds of structural disorder can be seen in Fig. 1.1

\subsubsection{Defects in materials}

The simplest type of defect that can be encountered in a crystal is a point defect. More complex defects with higher dimensionality such as line defects (dislocations) and planar defects (grain boundaries and stacking faults) can be present as well, but they are more difficult to model in first principle calculations, although very important for, e.g., mechanical properties of real solids; there are also concerns regarding the thermodynamic stability of some of these extended defects [6].

Point defects can be either intrinsic or extrinsic: intrinsic defects are vacancies and self-interstitials, which occur in pure materials, whereas extrinsic defects are foreign atoms present in the material which are not related to the compound itself.

A vacancy (Fig. 1.1a) is a vacant atomic site in the crystal lattice; the missing atom can end up in an interstitial position (in a pocket between regular sites of the lattice), or, for example, migrate to the surface. It is currently well known that important physical effects, such as mass transport, are governed by these type of defects, therefore it is very important to investigate and acquire a detailed understanding of how vacancies and self-interstitials are formed and diffuse in the crystal. Moreover, they are thermodynamic defects, i.e. they appear at finite 


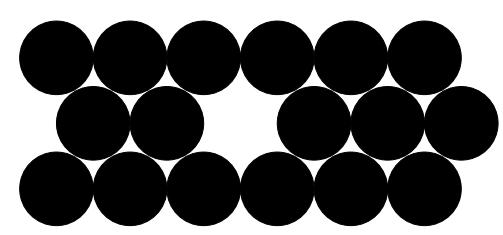

(a)

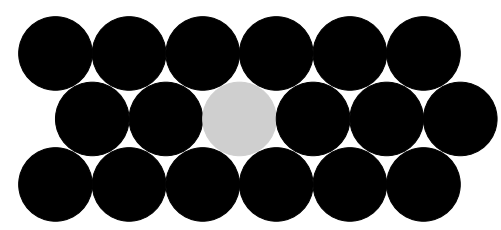

(c)

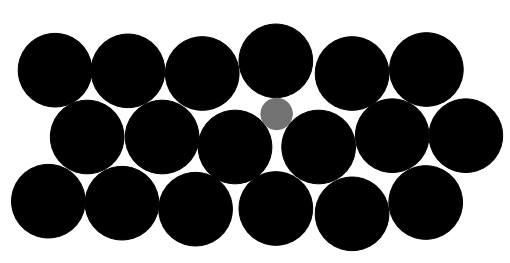

(b)

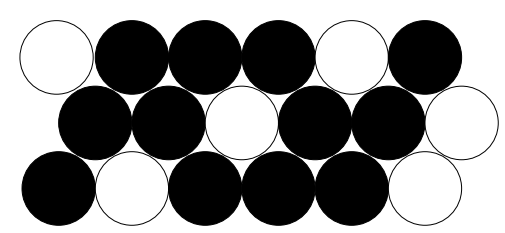

(d)

Figure 1.1: Schematic 2D descriptions of different types of disorder: a vacancy (a) and an extrinsic interstitial atom (b) in an otherwise perfect lattice, a substitutional extrinsic atom (c), and a random substitutional alloy (d).

temperature and they are stable because they increase the entropy of the system. The equilibrium concentration of vacancies $c_{\mathrm{vac}}$ at temperature $T$ can be easily calculated with a simple thermodynamic model that takes into account the configurational entropy arising from the introduction of vacant sites in a crystal, and this can be expressed as:

$$
c_{\mathrm{vac}}=\exp \left(-\frac{E_{\mathrm{vac}}^{f}}{k_{B} T}\right) .
$$

Here, $E_{\mathrm{vac}}^{f}$ is the vacancy formation energy, and $k_{B}$ is the Boltzmann constant. This model assumes that vacancies are very diluted in the system (no conglomeration of vacancies), and it does not take into account any effect of lattice vibrations. In order to be more accurate, one should take in the exponent the full Gibbs free energy, rather than the simple energy, to obtain a concentration with inclusion of temperature effects. Moreover, the Gibbs free energy is affected by vibrations as well: as it has been shown recently by Glensk et al. [7], the vacancy concentration deviates from the assumed Arrhenius law because of local anharmonicity of atomic vibrations into the vacancy. In addition, in a real sample vacancy concentrations 
can be higher than the equilibrium values for kinetic reasons related to the synthesis process. Self-interstitial atoms generally show a much higher formation energy compared to vacancies, so in absence of particular conditions such as energetic irradiation [8], they can in many cases be neglected.

Extrinsic defects are important as well: they can determine several properties of a materials, such as optical (e.g., impurities in diamonds [9] determine the color of the crystal), mechanical (as an example, hydrogen embrittlement in steels [10]), and electronic (doping of semiconductors [6]). When accidental, these defects occur either because in laboratory conditions it is impossible to maintain the environment free from any impurity during the growth of a crystal, or just by exposure of the material to atmosphere and consequent diffusion in the bulk. However, the amount of defects can be very small if the conditions are well controlled. As an example, in GaAs thin films grown with molecular beam epitaxy the concentration of impurities can be as low as 1 impurity every $10^{9}$ atoms [11]).

Experimentally, it can be difficult to measure the concentration of defects in a material. The concentration of thermal vacancies is in general quite low (for a low formation energy of $0.5 \mathrm{eV}$ at $500 \mathrm{~K}$, the equilibrium concentration is one vacancy every $\sim 10^{5}$ atoms), and at low temperatures equilibrium is reached slowly; since the concentration increases with temperature, this quantity can be measured only at high temperatures.

\section{Diffusion}

As previously mentioned, vacancies and interstitials are known to be the main protagonist of self-diffusion in close-packed materials. In order for diffusion to occur, the diffusing atom needs in general to overcome an energy barrier. This is a probabilistic process: atoms vibrating at a certain temperature have a non-zero probability to perform a jump from a site to another, and this probability will be higher if the kinetic energy of the atoms is higher. In principle, also exchange between two atoms in equilibrium positions can occur, however this mechanism has in ordinary conditions a much higher activation energy.

Diffusion through a vacant site occurs with a simple mechanism: an atom neighbor to the vacancy can overcome the energy barrier and jump onto the vacant site. In general, an atom will perform many attempts to jump, and in rare events it will manage to diffuse from one site to the other. The temperature dependence of the jump rate is in general modeled within a rare events theory through the calculation of the energy barrier, i.e. the difference in energy between the diffusing atom in equilibrium position and at the transition state. The jump rate $\Gamma$ follows then Arrhenius law:

$$
\Gamma(T)=A \exp \left(-\frac{E_{b}}{k_{B} T}\right),
$$

where $A$ is the attempt frequency (related to vibrations in the crystal), and $E_{b}$ is the energy barrier. In principle, the attempt frequency depends on temperature as well, however often it is considered constant. This law in general breaks down 
at temperatures close to melting because of thermal expansion and anharmonic lattice vibrations, leading to deviations to the expected behavior.

For what concerns migration of interstitial atoms (both self-interstitials and extrinsic ones, Fig. 1.1b), if the concentration of vacancies is not particularly high, they will diffuse throughout the crystal jumping between interstitial sites. This might be easy for a small foreign atom or for open structures, however in general one can think that this is not the main process governing mass transport.

More complex mechanisms can be involved as well in particular materials: it has been shown, for example, that in $\mathrm{NiAl}$ alloys a complex 4-stage mechanism that involves the exchange of different atomic species neighboring a vacancy [12] is the process governing mass transport.

\subsubsection{Alloys}

Alloys are a class of material where different metallic elements are mixed together. In principle, restrictions on the number of different elements that can be mixed is given by the periodic table, but only thermodynamics can say if a multicomponent alloy is stable or not. Alloys can be divided in two categories: ordered and disordered. Ordered alloys show a Bravais lattice with a basis, so that periodicity throughout the crystal is preserved. Disordered alloys (schematically represented in Fig. 1.1d), on the contrary, consists of a solid solution of elements without longrange order in the lattice, where every point in space is different from any other. However, average properties retain the lattice symmetry, therefore experimentally these are seen as periodic structures. A perfect example of these two types of alloys is beta brass, which is a $\mathrm{Cu}-\mathrm{Zn}$ alloy with roughly equal content of the two elements: at low temperatures, it has an ordered structure, but it undergoes a transition to a disordered alloy at high temperature. The order-disorder transition is driven by entropy: a disordered structure, although it might have a higher energy compared to the ordered structure, has a certain degree of configurational entropy; at high temperature, the entropic contribution can win over the energy, and disorder becomes more favorable.

The elements forming an alloy need not to be all metals: the best example of this is steel, which is a solid solution of $\mathrm{C}$ in Fe. Here, the $\mathrm{C}$ atoms sit in interstitial positions in the Fe lattice. For what concerns ferritic steel, i.e. C interstitials in bcc Fe, the solubility of $\mathrm{C}$ is so low that often these are modeled as point defects in the lattice.

The effect of entropy on structural stability is extremized in the so-called highentropy alloys [13], which are part of the larger class of multiprincipal component alloys, where 5 or more elements are mixed together to form a solid solution. In these materials, single-phase solid solutions can be stabilized at finite temperature by the high configurational entropy. In order to be thermodynamically stable, the Gibbs free energy of all possible competing phases has to be higher than that of the high entropy alloy. 


\subsection{Magnetism in materials}

Magnetism is a physical phenomenon that has been known since the early stages of civilization. Signs of the development of compasses can be traced back to 300-200 B.C. in China [14], if not even earlier in pre-Columbian American civilizations [15]. However, understanding of this effect has come only in the past two centuries. We now know that magnetism is connected to electricity through Maxwell's equations and relativistic theory, and magnetism in solids arises from electrons and their intrinsic spin angular momentum. A fully classical theory of magnetism in condensed matter predicts that a permanent magnetization is not possible [16], and only by accounting for the electron spin, a pure quantum effect, one can explain this phenomenon.

Isolated atoms always display interaction with a magnetic field, due to the electrons they possess. This interaction can be diamagnetic (the atomic magnetic moment opposes the field) or paramagnetic (viceversa). All substances show at least a component of diamagnetism, which is related to electrons that are paired in a single orbital with opposite spins. When an atom has unpaired electrons, instead, it can show paramagnetism.

Going to solids, things get more complicated because electrons on different atoms interact with each other, so that it is rare to find systems displaying longrange magnetic properties. Solids showing ordered magnetic structures are generally made of $3 \mathrm{~d}$ or $4 \mathrm{f}$ elements because the electrons in these orbitals are more localized compared to others.

A theory that would completely describe these phenomena should take into account the quantum many-body effects involved in the problem; however also semi-classical theories which consider the magnetic moments localized on atoms have helped in understanding the physics of these systems. Moreover, several properties of materials which needs accounting for many atoms can be calculated only within semi-classical models, being these computationally much cheaper than many-body methods. It is therefore important to continuously develop these models in order to represent reality as well as possible, benchmark the relevance of the approximations and provide predictions for unstudied systems.

Two models have been employed to understand the behavior of magnetic materials: the localized moments and itinerant electrons models. The former is a semi-classical model connected to the Heisenberg Hamiltonian (see Sec. 2.4.1) and describes fairly well insulators which show robust localized moments, whereas the latter is based on Stoner band theory of electrons. However, these are radicalizations of reality, and most systems show intermediate behaviors: as an example, bcc Fe is a metal, nonetheless it can be often described as a localized moments ferromagnet.

In the following, I will describe how temperature influences the magnetic properties of materials, and to do this we need to start with the ground state of a magnetic material. 


\subsubsection{Low temperature ordered structures}

Many types of magnetic order can be encountered in solids. The first type of order that comes to mind is ferromagnetism: in this structure, considering a localized moments picture, the moments on magnetic atoms are all aligned in the same direction, leading to domains with non-zero magnetization of the material also in absence of an applied magnetic field. This happens in these materials because the interaction between neighboring magnetic moments (the exchange interaction) favors parallel alignment. The most known elemental magnets ( $\mathrm{Fe}, \mathrm{Ni}, \mathrm{Co}$, and $\mathrm{Gd}$ ) are all ferromagnets in their ground state.

On the opposite, some materials show antiferromagnetic (AFM) behavior: the moments on neighboring atoms are anti-parallel, so that on a macroscopic scale no magnetization is observed. Antiferromagnetism is usually more complex than ferromagnetism, since in a material it can happen that moments on a certain crystallographic plane are aligned parallel to each other, whereas on another they are anti-parallel. Some structures display also frustration of the moments: as an example, if three atoms are arranged in a triangular structure and they interact antiferromagnetically with each other, there is no way to dispose the moments so that the interaction is minimal for all of them [17, 18]. This can as well lead to noncollinear ordering of the magnetic moments [18].

In between, some materials display what is known as ferrimagnetism, which consists of moments with different sizes that interacts antiferromagnetically, so that a net magnetization is observable. The first compound employed as a magnet [14], magnetite $\left(\mathrm{Fe}_{3} \mathrm{O}_{4}\right)$, is actually ferrimagnetic [19].

Other more exotic ground state structures such as spin-waves or spin glasses can appear, however what is relevant to this thesis is that temperature will always tend to disorder the direction of the moments with respect to each other because of increased entropy.

\subsubsection{Thermal excitations and the paramagnetic phase}

At low temperatures, magnetic thermal excitations display a behavior similar to phonons: quasiparticles named magnons consisting of spin-waves are represented in a semi-classical picture tilting slightly the moments on each atom according to the wave-vector of that particular excitation. Magnons, in analogy to phonons, show dispersion relations that connects the frequency with the wavevector of a particular mode. Increasing the temperature further, more and more magnons are created. At a certain temperature (called Curie or Néel temperature for ferromagnets and antiferromagnets, respectively) long range order is lost and the magnetization of the material goes to zero. Looking at the atomic scale, in most materials the magnetic moments are still present, but they become disordered leading to zero macroscopic magnetization. Above the transition temperature, the moments still display a certain degree of short range order that does not give rise to any macroscopic magnetization.

In the Heisenberg picture of magnetism, the disordering of the moments is explained with transversal excitations. These are due to the competition between exchange interactions and temperature: the stronger is the magnetic interaction, 
the more difficult will be to disorder the moments, and therefore the higher the temperature for the order-disorder transition.

\subsubsection{Itinerant vs localized moments models}

As previously mentioned, the two models that have been employed to understand solid state magnetism are the localized moments and the itinerant electron models.

The localized moments model assumes that every magnetic atom in the solid possesses a magnetic moment which does not change in magnitude with temperature. The interaction with other moments is usually described with a Heisenberg Hamiltonian, which in its simplest form reads:

$$
H=-\sum_{i} \sum_{j \neq i} J_{i j} \mathbf{e}_{i} \cdot \mathbf{e}_{j}
$$

Here, $J_{i j}$ is the exchange interaction between moment $i$ and $j, \mathbf{e}_{i}$ is the direction of moment $i$, and the sum runs over all moment pairs in the system. In the ferromagnetic (FM) state, all moments are parallel to each other, so that $\mathbf{e}_{i} \cdot \mathbf{e}_{j}=1$ for every $i$ and $j$; in an idealized paramagnetic (PM) state at infinite temperature, the average scalar product between the directions of the moments is $\left\langle\mathbf{e}_{i} \cdot \mathbf{e}_{j}\right\rangle=0$. In this model, it is assumed that the exchange interactions between two atoms depends only on their coordination and they do not depend on the surrounding environment; also, the localized moments are supposed to have a well defined and constant magnitude.

The itinerant electrons model, also known as Stoner model, considers the electrons in bands and tries to describe ferromagnetism in metals. In this model, the magnetization of the solid (molecular field) splits the electronic bands in spin-up and spin-down channels as described by Pauli paramagnetism, where the molecular field is due to the same spin-polarization of the bands in a chicken-and-egg frame. In particular, it can be shown that a material will show spontaneous ferromagnetism if $U g\left(E_{F}\right) \geq 1$, where $U$ is the Coulomb energy (i.e., the gain in energy due to spin-polarization) and $g\left(E_{F}\right)$ is the density of states (DOS) at the Fermi level. This is known as the Stoner criterion, and it entails that for a metal to be ferromagnetic, it needs to have a large DOS at the Fermi level and strong Coulomb energy (which gives exchange and, therefore, the molecular field), leading to different energies for spin-up and spin-down electronic bands. If the Stoner parameter $U g\left(E_{F}\right)<1$ but still large, the metal shows enhanced Pauli susceptibility, i.e. its answer to magnetic fields is stronger than for regular metals. This is the case of $\mathrm{Pd}$ and $\mathrm{Pt}$, which are almost ferromagnets [16].

As it can be understood from this discussion, the two models describe quite different situations, and in reality most materials behave somewhere in between these two models. As an example, the first model cannot really explain why the size of the local moments in bcc Fe is $2.2 \mu_{B}$ or longitudinal excitations of the moments, whereas the second one heavily overestimates $\mathrm{T}_{C}$ of magnetic materials, predicting that they become nonmagnetic (i.e., no local moment is preserved). 
CHAPTER 2

\section{Theoretical methods}

In this chapter, the methods employed in this work will be presented. I start from the fundamentals of Density functional theory (DFT), then move to important concepts in thermodynamics simulations, and finally address the description of structural and magnetic disorder in ab initio simulations.

\subsection{Density functional theory}

Regular matter is made of atoms, which follow the laws of quantum mechanics. In principle, we have the theoretical framework to calculate and predict any property [20] of atoms, molecules and materials by solving the Scrödinger equation. However, this is a formidable task and in practice it is not possible to do it exactly. For this reason, we need to come up with methods that allow to find solutions in a reasonable time. Since the advent of DFT and supercomputing, this challenge has become more realistic to be undertaken.

DFT, which is in principle exact but in practice involves the use of approximations, tries to solve the Schrödinger (or Dirac) equation avoiding its intrinsic many-body complications. In the past years, DFT has been proven to be very powerful in the prediction and explanation of properties of many materials [21].

In the first three sections I will discuss DFT without accounting for the electron spin to make the notation easier, however in Sec. 2.1.4 I will explain how the theory is complemented with spin-polarization. In addition, for fermions one needs to solve the relativistic Dirac equation. In the calculations performed in this work, a scalar relativistic form of this equation is solved, but for sake of clarity in the following chapter I will use the Schrödinger equation. 


\subsubsection{The Schrödinger equation}

Matter is made of atoms which compose of nuclei and electrons, that are quantum mechanical objects. To derive any property of such a system, one needs to solve the time dependent Schrödinger equation for all the atoms in the system under investigation:

$$
i \hbar \frac{\partial}{\partial t} \Psi\left(\left\{\mathbf{R}_{I}\right\},\left\{\mathbf{r}_{i}\right\}\right)=\hat{H} \Psi\left(\left\{\mathbf{R}_{I}\right\},\left\{\mathbf{r}_{i}\right\}\right),
$$

where $\Psi$ is the many-body wavefunction for the whole system containing nuclei and electrons at positions $\mathbf{R}_{\mathbf{I}}$ and $\mathbf{r}_{\mathbf{i}}$, respectively, and $\hat{H}$ is the Hamiltonian for the system (in atomic units):

$$
\begin{aligned}
\hat{H}=- & \frac{1}{2} \sum_{i=1}^{n} \nabla_{i}^{2}-\frac{1}{2} \sum_{I=1}^{N} \frac{1}{M_{I}} \nabla_{I}^{2}-\sum_{i, I} \frac{Z_{I}}{\left|\mathbf{r}_{\mathbf{i}}-\mathbf{R}_{\mathbf{I}}\right|}+\frac{1}{2} \sum_{i \neq j} \frac{1}{\left|\mathbf{r}_{\mathbf{i}}-\mathbf{r}_{\mathbf{j}}\right|}+ \\
& +\frac{1}{2} \sum_{I \neq J} \frac{Z_{I} Z_{J}}{\left|\mathbf{R}_{\mathbf{I}}-\mathbf{R}_{\mathbf{J}}\right|}+\hat{V}(\mathbf{r}, t) .
\end{aligned}
$$

In the Hamiltonian we find the kinetic energy for electrons (lowercase letters) and for the nuclei (capital letters), the attractive interaction between electrons and nuclei, and the electron-electron and nucleus-nucleus repulsion, respectively. The last term is a possible external potential, which can in general depend on space and time. $M_{I}$ and $Z_{I}$ are mass and charge of nucleus $I$, respectively.

If there is no explicit dependence on time in the Hamiltonian, the equation can be separated and one obtains the time-independent Schrödinger equation:

$$
\hat{H} \Psi=E \Psi,
$$

where $E$ is the energy of the system. This equation can be solved analytically only for the $\mathrm{H}$ atom, which has one proton and one electron, and in no way it can be solved analytically for a real solid.

In order to simplify the problem, one needs to employ approximations. The first approximation that can be made is the so-called Born-Oppenheimer approximation: since the mass of the electrons $m_{e}$ is much smaller than the mass of the nuclei $M_{I}$, then the nuclei are seen as frozen by the electrons and, as the nuclei moves, the electrons remain in the same state. In this way, the wavefunction can be separated in two contributions $\Psi\left(\left\{\mathbf{R}_{I}\right\},\left\{\mathbf{r}_{i}\right\}\right)=\Phi\left(\left\{\mathbf{R}_{I}\right\}\right) \psi\left(\left\{\mathbf{r}_{i}\right\}\right)$, where I have assumed that the time-dependent part has already been separated. The electronic wavefunction $\psi\left(\left\{\mathbf{r}_{i}\right\}\right)$ depends parametrically on the nuclear positions. It is also assumed that the electronic states are not too close to each other, so that perturbations due to nuclear kinetic energy do not affect the electron system [21].

From here, one further approximation can be to consider the nuclei as classical particles, so that the quantum problem has to be solved "only" for the electronic system.

For what concerns the solution of the electronic problem for a crystalline solid, Bloch's theorem comes in help at this point, allowing to solve the problem for 
an infinite periodic solid taking into account only the primitive cell of the lattice. This theorem states that, for an electron in a periodic potential (here due to the nuclei), its wavefunction $\psi$ can be expressed as:

$$
\psi(\mathbf{r})=e^{i \mathbf{k} \cdot \mathbf{r}} u_{l \mathbf{k}}(\mathbf{r}),
$$

where $\mathbf{k}$ is a reciprocal lattice vector, $\mathbf{r}$ is the position, $l$ is the quantum number (band index) of the wavefunction, and $u_{l \mathbf{k}}(\mathbf{r})$ is a function with the periodicity of the potential.

Thomas and Fermi in 1927 separately proposed a scheme that enables to solve approximately the Schrödinger equation. This is done by changing the focus of the problem from the electronic wavefunctions $\psi\left(\mathbf{r}_{i}\right)$ to the electronic density $n(\mathbf{r})$ in the solid at position $\mathbf{r}$. In this model, the electron density is assumed to be locally constant and the kinetic energy is taken as the kinetic energy of a homogeneous electron gas. The model fails quite badly to predict properties of molecules and materials, but it was a first step towards the birth of DFT, introducing the fundamental concept of electron density as the important physical quantity to calculate.

\subsubsection{The Hohenberg-Kohn theorems and the Kohn-Sham ansatz}

In 1964, Hohenberg and Kohn published an article [1] in which they proved that, for an electronic system under the action of an external potential, there is a oneto-one relation between the potential and the electron density, so that knowledge of one of the two gives immediately the other quantity. In addition, the energy of the system is a universal functional of the electron density, which can be found variationally. The power of these theorems is that they are disarmingly simple to prove, and they give a strong and firm theoretical basis for all the calculations performed within the DFT framework.

Unfortunately, the Hohenberg-Kohn theorems do not provide any scheme to find the universal functional of the density, and this is were the actual approximations come into play. In general, the energy functional can be written as:

$$
E[n]=T[n]+E_{\text {int }}[n]+\int \mathrm{d} \mathbf{r} V_{\text {ext }}(\mathbf{r}) n(\mathbf{r})+E_{I I},
$$

where $T$ is the kinetic energy of the electrons, $E_{\text {int }}$ is the electron-electron interaction, the integral (extended over all space) is the interaction of the electrons with the external potential $V_{\text {ext }}$ due to the nuclei, and $E_{I I}$ is the interaction between nuclei. This functional is not known in general, so that it is practically useless as it is.

Kohn and Sham suggested a practical scheme [2] that uses the ansatz that we can calculate the properties of the real system by taking into account an auxiliary system of noninteracting electrons displaying the same electron density as the

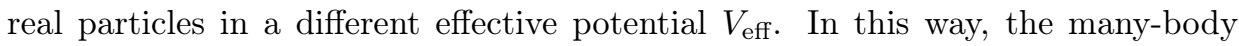
wavefunction for all the electrons can be replaced by independent single-particle wavefunctions $\psi_{i}$, and one needs to solve a system of coupled equations: 


$$
\left(-\frac{1}{2} \nabla_{i}^{2}+V_{\text {eff }}\right) \psi_{i}=\varepsilon_{i} \psi_{i}
$$

where the single-particle wavefunctions are related to the electron density by:

$$
n(\mathbf{r})=\sum_{i}\left|\psi_{i}(\mathbf{r})\right|^{2}
$$

From here, one can derive an expression of the energy functional for the whole system:

$$
E[n]=T_{S}[n]+\int \mathrm{d} \mathbf{r} V_{\text {ext }}(\mathbf{r}) n(\mathbf{r})+E_{\text {Hartree }}[n]+E_{I I}+E_{\mathrm{xc}}[n],
$$

with

$$
\begin{gathered}
T_{S}[n]=\frac{1}{2} \sum_{i}\left|\nabla_{i} \psi_{i}\right|^{2}, \\
E_{\text {Hartree }}[n]=\iint \mathrm{d} \mathbf{r} \mathrm{d} \mathbf{r}^{\prime} \frac{n(\mathbf{r}) n\left(\mathbf{r}^{\prime}\right)}{\left|\mathbf{r}-\mathbf{r}^{\prime}\right|} .
\end{gathered}
$$

The functional now contains the kinetic energy of the independent electrons $T_{S}$, the classical interaction of the electron density with itself $\left(E_{\text {Hartree }}\right)$, the interaction of the density with the external potential, the interaction between nuclei, and a last term which is called exchange and correlation functional in which all the terms that are not included in the independent particles approximation are collected. In general, the first terms can be calculated exactly; the last one, on the contrary, is unknown and one needs to find a good approximation to the exchange and correlation energy in order to predict properties. The physical origin of this term is double: on the one hand, it takes into account the Pauli exclusion principle, which is not treated by the Hartree term (the so called exchange); on the other hand, it includes the Coulomb repulsion between electrons of opposite spin, which is partly due to the kinetic energy and partly to the Hartree term (correlation). Approximations to the exchange and correlation term will be treated in the next section.

As previously mentioned, this procedure to calculate the functional of the density is an ansatz: this means that there is no rigorous proof that a correspondence between the real interacting electron system and the auxiliary non-interacting system exist. However, the Kohn-Sham method has been widely used with success, and most of the times inaccuracies are due to the exchange and correlation term rather than on the whole framework, although some quantities are known to be out of reach for this scheme [21]. The success of this method is due to the fact that, in normal system, the exchange and correlation term is small, so that an approximation to it can still give good results. The power of the Kohn-Sham ansatz as compared to Thomas-Fermi theory is in the fact that the kinetic energy of the electrons is not approximated as the homogeneous electron gas at every point in space, rather is the kinetic energy of independent electrons on top of which one adds exchange and correlation. 
Methods to practically solve the Kohn-Sham equations rely on expansion of the wavefunctions $\psi_{i}$ in some basis set. In this work we have used plane-waves as basis since they are highly compatible with the periodic nature of crystalline solids and Bloch's theorem. In addition, in this work the projector augmented wave (PAW) method [22] has been used to represent the single-particle wavefunctions. In this method, space is separated in two regions: an augmentation region around nuclei, and an interstitial region in between the atoms. Plane waves are good to represent valence electrons in the interstitial region, but they are not good for describing the rapidly oscillating wavefunctions near the nuclei. In this region, an all-electron wavefunction must respect orthogonality with the core electrons. To do this, but at the same time use smoother wavefunctions in the calculations, the PAW method prescribes a transformation from the original wavefunctions to auxiliary ones, which are smooth in the augmentation region. Calculations are performed with these last functions, which coincides with the original ones in the interstitial region, and finally the results for the original wavefunctions are recovered by the inverse transformation.

\subsubsection{Exchange and correlation functional}

As previously mentioned, the exchange and correlation functional is the approximated term in DFT which determines an upper limit to the accuracy of calculations.

The first approximation developed for the exchange and correlation energy is the famous local density approximation (LDA). Within this approximation, the electron density at each point is assumed to have exchange and correlation energy equal to that of a homogeneous electron gas. This is expressed as:

$$
\begin{aligned}
E_{\mathrm{xc}}^{\mathrm{LDA}} & =\int \mathrm{d} \mathbf{r} n(\mathbf{r}) \epsilon_{\mathrm{xc}}^{\mathrm{hom}}(n(\mathbf{r})) \\
& =\int \mathrm{d} \mathbf{r} n(\mathbf{r})\left[\epsilon_{\mathrm{x}}^{\mathrm{hom}}(n(\mathbf{r}))+\epsilon_{\mathrm{c}}^{\mathrm{hom}}(n(\mathbf{r}))\right]
\end{aligned}
$$

The exchange energy for a homogeneous electron gas is here calculated analytically, whereas the correlation energy has to be calculated numerically with higher-level theory methods. The first quantitatively accurate calculation of $\epsilon_{\mathrm{c}}^{\text {hom }}$ was performed by Ceperley and Alder [23] with quantum Monte Carlo calculations.

Despite the crude approximation of considering exchange and correlation effects at each point of a solid as for a homogeneous electron gas, the LDA functional is often capable of capturing the physics of systems like nearly-free-electron metals, and it is still used nowadays in certain cases. What makes LDA able to capture at least trends in materials is the fact that it is based on a real electronic Hamiltonian: for this reason, it satisfies constraints regarding the properties of the exchange and correlation hole [21]. In addition, only the spherical average of the exchange and correlation hole enters the energy, so that detailed knowledge of its shape is not needed. 
From here, the obvious way to improve the exchange and correlation functional is to include the gradient of the electron density; however this has been more difficult than expected. The problem here is that gradients of the density can be very large in real materials, so that a simple power expansion breaks down. In order to be useful, a generalized gradient approximation (GGA) has to be used, imposing the expected behavior at large gradients. GGA functionals, in particular the formulation by Perdew, Burke, and Ernzerhof (PBE) [24], has lead to great improvement compared to LDA, especially in the calculation of equilibrium volumes and atomization energies of molecules [24]. GGAs are semi-local functionals, because they require the density in an infinitesimal neighborhood of $\mathbf{r}$ in order to get derivatives, in contrast to LDA which requires the density only at $\mathbf{r}$, therefore is local.

Since then, many research groups around the world have tried to improve further the exchange and correlation functionals, trying to satisfy more and more exact constraints, following a "Jacob's ladder" [25] of density functional approximations that tends to reach chemical accuracy $(1 \mathrm{kcal} / \mathrm{mol}=0.044 \mathrm{eV} /$ formula unit) [26]. The ladder starts from LDA, for which only the local density is taken into account, going to GGAs where also gradients of the density are included; the next rung is occupied by meta-GGAs, for which limits of the electron kinetic energy densities are also satisfied, and further on we find methods that include exact exchange and compatible or exact partial correlation.

The higher rungs of the ladder involve methods (like hybrid functionals or random phase approximation) that are too computationally expensive for regular calculations, and are in general used when very accurate results are needed or for properties that cannot be predicted with usual functionals (e.g., bandgaps). For what concerns meta-GGAs, they are comparable in terms of cost with semilocal functionals, but they are thought to describe better electron correlation because of the direct inclusion of the kinetic energy density in the functional. One of the most recent meta-GGAs that has been discussed quite widely is the strongly constrained and appropriately normed (SCAN) [27] functional, which is the only known meta-GGA that satisfies all the known constraints for this category.

LDA and GGAs are known to describe badly some $3 \mathrm{~d}$ and $4 \mathrm{f}$ elements in strongly correlated compounds because they are not able to reproduce the localization of these orbitals. A simple method that has been devised to make this problem less serious is the so called "DFT $+\mathrm{U}$ ", where an Hubbard U term is added in the energy functional for localized orbitals. The $\mathrm{U}$ term tends to localize electrons by adding an energy cost for an electron in an individually occupied state to jump onto another state which is already singly occupied, therefore keeping the electrons apart. The main effect of this term is to split the energy levels of these orbitals, and in general it is used to open an electronic bandgap in semiconductors or insulators that are predicted to be metals with conventional DFT.

\subsubsection{Spin-polarized DFT}

Of course, electrons are spin- $1 / 2$ particles, therefore all the equations previously mentioned should be reexpressed in order to include this property. The easiest 
way is just to separate the electron density in a spin-up and a spin-down electron density, namely:

$$
n(\mathbf{r})=n^{\uparrow}(\mathbf{r})+n^{\downarrow}(\mathbf{r})
$$

and all the equations are solved separately for the two spin-polarized densities. In general, this is needed only for magnetic materials.

If noncollinear magnetic moments are to be employed in the calculations, the electron density can be represented in terms of a spin vector at every point, and the single-particle Hamiltonian becomes a 2x2 matrix:

$$
H_{\mathrm{KS}}^{\alpha \beta}=-\frac{1}{2} \nabla^{2}+V_{\mathrm{eff}}^{\alpha \beta},
$$

where $\alpha$ and $\beta$ indicate the two eigenspinors for an electron. The only nondiagonal term in the effective potential is the exchange and correlation term. In calculations, this procedure is carried out by finding the local axis of spin quantization and then using the spin-polarized exchange and correlation energy for that axis.

In this work, a constrained formulation of DFT has been used in order to select the direction of the atomic magnetic moments. Constrained DFT consists in adding a Lagrange multiplier to the energy of the system, so that the solution obtained will minimize the energy subject to the constraint. The method used in this work to constrain the direction of the moments has been developed by Ma and Dudarev [28], in which a penalty energy $E_{S}^{p}$ at site $S$ is added to the DFT energy:

$$
E_{S}^{p}=\sum_{S} \lambda_{S}\left|\mathbf{M}_{S}^{F}\right|-\mathbf{e}_{S} \cdot \mathbf{M}_{S}^{F}
$$

where $\lambda_{S}$ is a Lagrange multiplier, $\mathbf{e}_{S}$ is a unit vector in the direction along which the moment has to be constrained, and $\mathbf{M}_{S}^{F}$ is the magnetic moment defined as:

$$
\mathbf{M}_{S}^{F}=\int_{\Omega_{S}} \mathrm{~d} \mathbf{r} \mathbf{m}(\mathbf{r}) F_{S}\left(\left|\mathbf{r}-\mathbf{r}_{S}\right|\right) .
$$

Here, $\mathbf{m}(\mathbf{r})$ is the magnetization density at point $\mathbf{r}, \Omega_{S}$ is the atomic sphere, and $F_{S}\left(\left|\mathbf{r}-\mathbf{r}_{S}\right|\right)$ is a function that decreases monotonically to zero towards the boundary. With this method, the direction of the moments can be constrained at will, and it can be improved by increasing further and further the value of the Lagrange multiplier $\lambda_{S}$. In addition, within this formulation it is less favorable for the magnetic moment to change sign maintaining the same direction, in contrast with other methods that do not penalize spin flips. It can also be shown that the obtained penalty energy tends to zero for increasing $\lambda_{S}$.

\subsection{Thermodynamic simulations}

DFT enables the calculation of the ground state energy and related properties of a system. Since it is a ground state method, in principle its results are only for zero 
temperature. It has been shown that the free energy of the electronic system can be calculated with the Mermin's functional [29] at a given temperature by including the proper smearing of the wavefunctions. However, to have the full free energy of the system, one needs to take into account contributions due to vibrations (and magnetism, if needed). Therefore, it is needed to complement DFT calculations with thermodynamic simulations that allow for the inclusion of temperature effects at every level. Moreover, many interesting properties are related to the system dynamics, so that one needs to be able to realistically simulate the movement of atoms, as well as magnetic and configurational degrees of freedom.

In computer simulations, it is usually easier to fix the number of atoms, the volume of the system and its temperature. Therefore, what we usually simulate is a canonical ensemble, so that the following discussion will focus on this.

As previously mentioned, in some cases we can consider atoms as classical particles, so that the infrastructure of classical thermodynamics can be applied. One of the central quantities in thermodynamics is the free energy: it is good to start the discussion on thermodynamic simulations from this.

\subsubsection{Free energy}

Given a system of $N$ particles with constant volume $V$ in contact with a heat bath at temperature $T$, from statistical mechanics and thermodynamics we know that we can define a free energy $F$ up to a constant value, which is given by:

$$
F=E-T S=-k_{B} T \log (Z),
$$

where $E$ is the energy of the system, $S$ is the entropy, $k_{B}$ is the Boltzmann's constant, and $Z$ is the partition function. The equilibrium state for such a system is the one with the lowest free energy. We know that all thermodynamic quantities can be derived from the knowledge of the partition function of a system, and therefore from knowledge of $F$. The canonical partition function is defined as:

$$
Z=\sum_{i} \exp \left(-\frac{E_{i}}{k_{B} T}\right)
$$

where the sum runs over all the possible microstates that the system can explore. This means that, in order to be able to say anything about the thermodynamics of a system, we need to be able to sample properly its phase space, i.e. the collection of all the accessible microstates of the system.

The calculation of the partition function is a formidable task, which can be sometimes performed analytically for simple model systems, but is typically not possible for realistic systems. What is usually done is to calculate the free energy applying some approximation. A common starting point is to decouple degrees of freedom that are relative to different time scales. As an example, for a magnetic material, it is often assumed that electronic degrees of freedom are much faster than magnetic ones, which are faster than vibrational ones, so that the free energy can be expressed as: 


$$
F(V, T)=E_{0 \mathrm{~K}}(V)+F^{\mathrm{el}}(V, T)+F^{\mathrm{vib}}(V, T)+F^{\mathrm{mag}}(V, T)+F^{\text {ad. coup. }}(V, T) .
$$

This separation of degrees of freedom allows one to calculate separately the differ-

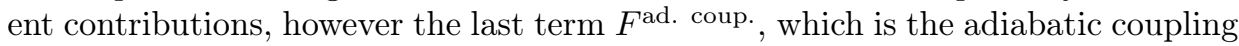
between the different degrees of freedom, can only be inferred from simulations that include all effects at the same time.

An example of such a calculation of the free energy of, for simplicity, a nonmagnetic system would start with the calculation of its ground state energy $\left(E_{0 \mathrm{~K}}(V)\right)$ with DFT, where also the effect of temperature on the electronic degrees of freedom can be included $\left(F^{\mathrm{el}}(V, T)\right)$; then, assuming harmonic behavior of lattice vibrations, one can calculate the phonon dispersion with, e. g., the finite displacement method, and from here the vibrational free energy $\left(F^{\mathrm{vib}}(V, T)\right)$ is obtained (for harmonic phonons, the vibrational free energy has an analytic expression, see e.g [6]). Of course, it is well known that the harmonic approximation does not work at every temperature since it does not explain thermal expansion, so what is often employed is the quasi-harmonic approximation, i.e. the phonon dispersion is calculated at different volumes that follow the thermal expansion of the system. If one wants to reduce approximations as much as possible, then thermodynamic simulations have to be performed in order to fully account for the different degrees of freedom and their adiabatic interplay, and to sample appropriately the phase space.

As earlier mentioned, here I focus on the canonical ensemble because it is the easiest to simulate. In an experiment, though, the volume is not a fixed quantity, rather the pressure $p$ is fixed. Therefore, in experiments the thermodynamic quantity of relevance is the Gibbs free energy $G$ :

$$
G=E-T S+p V=H-T S=F+p V,
$$

where $H=E+p V$ is the enthalpy. It is well known that the equilibrium state of a system at a certain temperature and pressure is the one with minimum Gibbs free energy, therefore if one wants to calculate equilibrium phases, then it is needed either to perform calculations at constant pressure, or to perform calculations in the canonical ensemble on a grid of temperatures and volumes so that the pressure can be calculated as:

$$
p=-\left(\frac{\partial F}{\partial V}\right)_{T, N},
$$

and consequently obtaining the Gibbs free energy.

So far, I have not discussed how one can actually calculate the full free energy of a system. As previously mentioned, the free energy is defined up to a constant, so that what matters in general are free energy differences. This fact is exploited in thermodynamic integration [30], in which the potential energy of a model system, for which the free energy is known, is coupled with the potential energy of the system through a parameter $\lambda$, so that performing proper phase space sampling and integrating over several values of $\lambda$ one can obtain the free energy difference 
between the model and the real system, and therefore the full free energy. This technique has been used, as an example, for calculating the free energy of metals $[7,31,32]$ from $0 \mathrm{~K}$ up to the melting point, as well as the vacancy formation free energy in the same systems.

The computational methods employed for phase space sampling are molecular dynamics (MD) and Monte Carlo (MC) simulations. The technique mainly used in this work is MD, which will be discussed in the next paragraph. For what concerns Monte Carlo simulations, the main idea is to explore the phase space with random jumps from one state to the other, and these jumps are either accepted or rejected depending on the energy of the state. In particular, for the Metropolis algorithm, if the new state has an energy lower than the previous, then the jump is accepted, whereas if the energy is higher, then the jump will be accepted if and only if the ratio between the Boltzmann factor of the new and the old state is larger than a random number uniformly taken in the range $[0,1]$.

Another important detail to take into account is how the energy of the system (both in MC and MD) is calculated. In general, for computational reasons, a model interatomic potential for the system under investigation is prepared and energies are calculated from this. These potentials allow to perform simulations with a huge number of atoms, however they lack in accuracy and transferability. Another way is to calculate the energy (and forces in MD) with DFT, making the method ab initio.

\subsubsection{Ab initio molecular dynamics}

Molecular dynamics is a technique that enables to sample phase space of a system by simulating the trajectory of the atoms in the system. It can be in general performed in different ensembles (microcanonical, canonical, and constant-pressure), and potential energy and forces can be calculated either with model potentials (classical MD), or with DFT (ab initio MD, AIMD). In both cases, the nuclei are considered as classical particles which follow Newton's equations of motion:

$$
\mathbf{R}_{I}(t+\mathrm{d} t)=\mathbf{R}_{I}(t)+\mathbf{v}_{I}(t) \mathrm{d} t+\frac{\mathbf{f}_{I}(t)}{2 M_{I}} \mathrm{~d} t^{2},
$$

where $\mathbf{R}_{I}(t)$ and $\mathbf{v}_{I}(t)$ are the position and velocity of atom $I$ with mass $M_{I}$, and $\mathbf{f}_{I}(t)$ is the force acting on it at time $t$. This equation is not actually used in MD simulations because it is not stable, so that other algorithms are employed (such as the Verlet or the velocity-Verlet algorithm, see e.g. [30] for a collection of methods). A central quantity in this equation is the timestep $\mathrm{d} t$, which has to be chosen carefully in order to obtain meaningful simulations (a typical value in AIMD is $1 \mathrm{fs}$ ).

Forces in AIMD are calculated according to the Hellmann-Feynman theorem:

$$
\mathbf{f}_{I}=-\left\langle\frac{\partial \hat{H}}{\partial \mathbf{R}_{I}}\right\rangle,
$$

where $\hat{H}$ is the Hamiltonian of the system, and \langle\rangle indicates the expectation value. 
AIMD gives a very accurate description of the dynamics of the system, however it is very computationally intensive so that only small systems can be simulated (commonly $\sim 100-200$ atoms, at best $\sim 1000)$ and for short timescales $(\sim 1$ ns). If larger sizes are needed, one can go to classical potentials that can be based on analytical models or fitted to ab initio results in order to perform larger scale simulations. In the last years, machine learning potentials have been introduced in this field, which are very promising for performing large simulations at low computational costs retaining almost DFT accuracy (see, e.g., [33] for a review).

For calculations in the canonical ensemble, the system needs to be coupled with a thermostat which maintains the temperature of the system roughly constant (the amplitude of thermal fluctuations depend on the size of the system). Several thermostats are available: the simplest method consists in simply rescaling the velocity of the atoms in order to meet the required temperature. Other methods consists in coupling the system with a thermostat that randomly pushes the atoms (Andersen and Langevin thermostats), resulting in a very good sampling of the phase space but with possible destruction of the system physical dynamics. The NoséHoover thermostat, instead, introduces a fictitious system through an extended Lagrangian formulation which keeps the real system at the desired temperature. This is the thermostat that influences the least the dynamics of the system. It is also interesting to discuss briefly the Langevin thermostat: this method was developed for dynamics simulations of liquids, but it has been then employed for simulations of solids. In this method (also known as Langevin dynamics), the equations of motions are modified such that:

$$
\dot{\mathbf{p}}_{I}=\mathbf{f}_{I}-\gamma_{I} \mathbf{p}_{I}+\overline{\mathbf{f}}_{I} .
$$

Here, $\mathbf{p}_{I}$ is the momentum of atom $I, \dot{\mathbf{p}}_{I}$ its time derivative, $\gamma_{I}$ is a friction coefficient (in units of inverse time), and $\overline{\mathbf{f}}_{I}$ is a random force related to $\gamma_{I}$. The parameter $\gamma_{I}$ determines how often the system is "kicked" by the random force, so that for large values of this parameter one can employ large timesteps, obtaining a very good and fast phase-space sampling, at the expense of unrealistic dynamics (although it is still possible to recover the proper dynamics by decreasing $\gamma_{I}$ ).

\subsubsection{Nonequilibrium molecular dynamics - Color Diffusion algorithm}

Direct MD simulations can be employed in the study of diffusion or other physical phenomena that involve dynamics in order to obtain the rate and mechanism of the property under investigation; unfortunately, for rare events it might not give a good statistic of the process. Many methods have been developed in the past years in order to overcome this obstacle, and in this work a revisited version [34] of the color diffusion (CD) algorithm [35] has been employed.

The CD algorithm is part of the broader class of nonequilibrium MD (NEMD) methods, which in general consists in applying a force on the system in order to accelerate the physical process of interest. As an example, if one wants to accelerate the vacancy-mediated diffusion of an atom in a solid, the CD algorithm in the revisited version consists simply in applying a force on one of the neighbors 


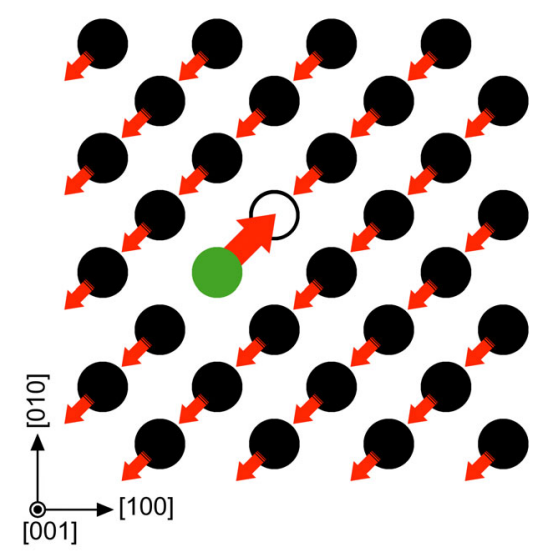

(a)

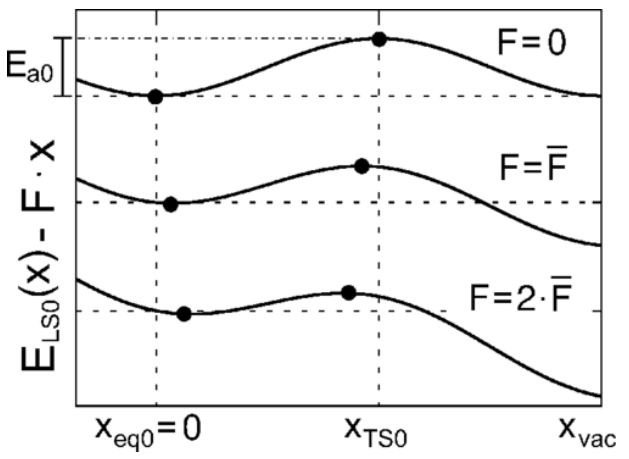

(b)

Figure 2.1: a) Principle of the color diffusion algorithm: the colored atom (green) is pushed towards the vacancy (white) with a force $F$. An opposite force of intensity $F /(N-1)$, with $N-1$ the number of atoms in the simulation box without counting the colored atom, is applied to all the other atoms in order to maintain the system in mechanical equilibrium. Reprinted figure with permission from D. Gambino, D. G. Sangiovanni, B. Alling, and I. A. Abrikosov, Physical Review B 96, 104306 (2017). Copyright 2017 by the American Physical Society. b) Effect of the force on the energy landscape, with $x_{\mathrm{eq}}$ the equilibrium position of the colored atom, and $x_{\mathrm{TS}}$ the transition state. Reprinted figure with permission from D. G. Sangiovanni, O. Hellman, B. Alling, and I. A. Abrikosov, Physical Review B 93, 094305 (2016). Copyright 2016 by the American Physical Society https://doi.org/10.1103/PhysRevB.93.094305.

(the colored atom) to the vacancy so that it will jump earlier than in equilibrium conditions (see Fig. 2.1a). The nonequilibrium jump rate is calculated for different intensities of the force field, and the equilibrium jump rate is then recovered by extrapolation to vanishing force. In order to maintain the system in mechanical equilibrium, a rescaled opposite force is applied on all the other atoms so that the resultant of the forces in the system is zero. The non-colored atoms are also coupled to a strong thermostat in order to dissipate the energy increase due to the external work.

The main difference between the original and the revisited version of the CD algorithm is that in the former the force fields employed are small in order to stay in the linear regime, whereas in the latter an extension to higher force fields has been made. It has been shown in [34] that, assuming a sinusoidal behavior of the energy landscape, the nonequilibrium jump rate $\Gamma$ as a function of the force field intensity $F$ and temperature $T$ can be expressed as:

$$
\Gamma(F, T)=\Gamma_{E}(T) \exp \left[\frac{x_{T S 0}(T)}{k_{B} T} F-\alpha(T) F^{2}\right]
$$


with $x_{T S 0}(T)$ the equilibrium transition state position at temperature $T$ (taking as 0 the equilibrium position of the atom, see Fig. 2.1b), $\alpha$ a fitting parameter, and $\Gamma_{E}(T)$ the equilibrium jump rate.

The effect of the force field is to tilt the energy landscape as shown in Fig. 2.1b. From here, one can see that the equilibrium position of the colored atom and the transition state get closer and closer for increasing force field intensities. At $F_{\max }$, transition state and equilibrium position coincide and the system becomes dynamically unstable, therefore this is the maximum intensity that can be used in this scheme. In fact, it has been observed that the fitting works best with force field intensities up to $F=0.75 F_{\max }$.

By taking the logarithm of equation 2.24 the relation between jump rate and force field becomes:

$$
\log \Gamma(F, T)=\log \Gamma_{E}(T)+\frac{x_{T S 0}(T)}{k_{B} T} F-\alpha(T) F^{2},
$$

i.e. the equilibrium jump rate can be found from a quadratic fitting of the nonequilibrium ones as a function of force field intensity. In order to reduce the number of force fields needed for the extrapolation, one can fix the value of the transition state position to the one obtained, e.g., with nudged elastic band (NEB) calculations taking into account thermal expansion.

So far, I have not explained how the nonequilibrium jump rate is practically calculated at a certain temperature and force field intensity. What is done here is to perform several NEMD calculations at temperature $T$ and force field intensity $F$ starting from uncorrelated initial configurations. Since the process is a rare event process, it can happen that in a simulation the jump of the colored atom is never observed: for this reason, the different runs are stopped either when the colored atom jumps, or when at least $75 \%$ of the simulations have lead to a jump of the colored atom. The jump times are then fitted according to a suitable probability distribution. For random processes like vacancy-mediated diffusion, the jumps are assumed to be in general independent and uncorrelated. The probability density function (PDF) that describes the time intervening between a jump and the successive one is the exponential PDF [36]:

$$
P D F_{k}(t)=k e^{-k t},
$$

where $t$ is time, and $k$ is the rate of the process, so that the average time of occurrence can be simply calculated as $\langle t\rangle=k^{-1}$. However, the exponential distribution is a special case of a family of PDF called the Gamma distribution, for which the PDF is expressed as:

$$
P D F_{\lambda, \theta}(t)=\frac{t^{\lambda-1} e^{-t / \theta}}{\theta^{\lambda} \Gamma(\lambda)},
$$

where $\lambda$ is the shape parameter, $\theta$ is the scale parameter, and $\Gamma(\lambda)=\int_{0}^{+\infty} x^{\lambda-1} e^{-x} \mathrm{~d} x$ is the Gamma function. The average occurrence time is here $\langle t\rangle=\lambda \theta$. Different types of Gamma distributions can be seen in Fig. 2.2. In this work, it has been observed that for low intensities of the force field, the distribution of jump times 


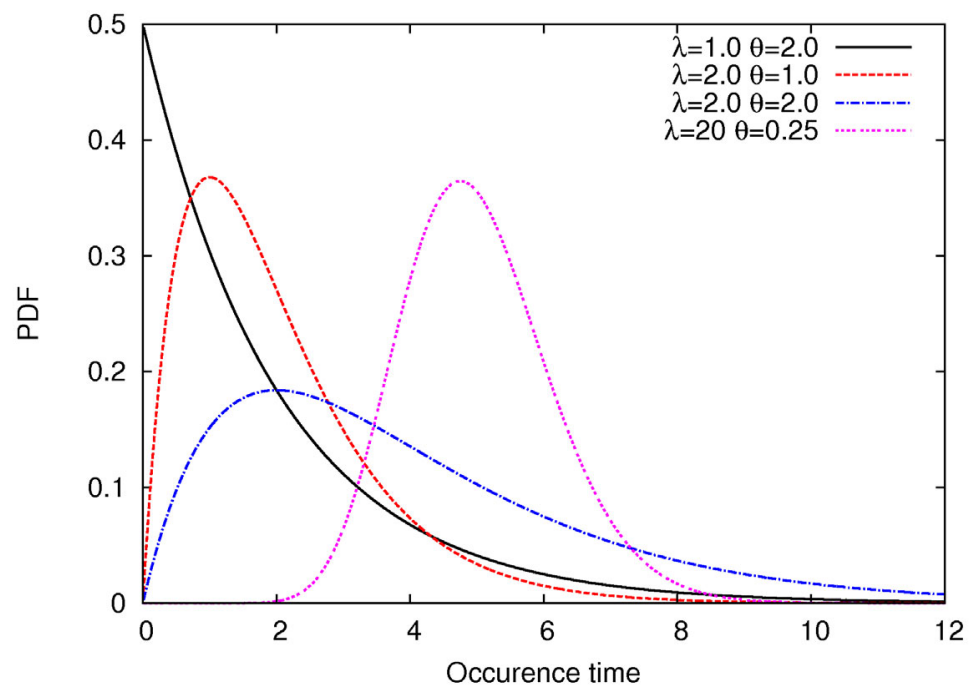

Figure 2.2: Examples of gamma distributions for different values of the shape $(\lambda)$ and scale $(\theta)$ parameters. For $\lambda=1$, the gamma distribution is reduced to an exponential distribution.

closely resembles an exponential distribution, whereas for high intensities of the force field the same distribution is more similar to a Gamma distribution with shape parameter $\lambda>1$, probably due to a correlation between the jump times triggered by the strong force fields.

Fitting the jump times obtained from NEMD simulations at a particular $F$ and $T$ with any of these two distributions gives a nonequilibrium jump rate with a well defined uncertainty, which can then be used in the extrapolation to zero force field with Eq. 2.25. It is important here to have well defined uncertainties for two reasons: first, the method is based on statistics, therefore uncertainties assess the precision of the calculated values; second, the physical process of diffusion is a probabilistic process, therefore uncertainties are inherently related to experimental results as well.

\subsection{Ab initio description of structural disorder}

The exponential increase of computational power of the last 30 years has enabled DFT calculations of systems with always growing size. This is very important for the proper description of point defects and substitutional disordered alloys from first principles, since for these type of problems the unit cell of a material cannot be used and one needs to resort to supercells of considerable size, unless mean-field methods such as the coherent potential approximation (CPA) are used. 


\subsubsection{Point defects}

As mentioned in Sec. 2.1.2, Bloch's theorem and periodic boundary conditions (PBC) enable the calculation of properties of a perfect infinite crystal by taking into account only the unit cell. If one wants to calculate the properties of a defect in the dilute limit (i.e., the concentration of defects is small), what can be done is to take a supercell made by the repetition of unit cells in all directions and introduce one defect in this simulation box. The validity of this method is ensured by the principle of "nearsightedness" [37], which states that a change in the potential at a point far away has a small effect on the region of interest. This seems to contradict quantum mechanics, for which eigenstates of a particle depend on the potential at any other point and on boundary conditions; however, for a manybody system, destructive interference between the wavefunctions of many particles reduces this nonlocal effect. An important thing to test therefore in calculations of defect properties is the size of the supercell: this means that the quantity of interest should be checked for convergence against the supercell size. At this point, one can assume that the point defect is not interacting anymore with its periodic images and the formation energy can be taken as the formation energy in the dilute limit.

It is important to consider as well the nature of the host material. For defects in metals, it is clear that only neutrally charged defects can be created. However, this is not the case for a semiconductor or an insulator, therefore one should take into account also this possibility in order to predict if the most stable defect is charged or neutral. In case of charged defects, careful convergence with supercell size is required since electrostatic interactions are long-ranged, and interaction with periodic images has to be avoided. Long-range interactions between defects might be present in metals as well: as an example, if magnetic impurities are placed in a metal, these can indirectly interact with each other through the conduction electrons of the host by spin-polarizing them. This coupling is known as Ruderman-Kittel-Kasuya-Yosida (RKKY) interaction [16], and in supercells calculations they need to be taken into account.

The formation energy of a defect $E_{\mathrm{d}}^{f}$ is defined with respect to the relevant chemical potentials, and it takes the general form (including the possibility of charged defects) $[38,39]$ :

$$
E_{\mathrm{d}}^{f}=E_{\mathrm{d}}-E_{\mathrm{bulk}}-\sum_{i} n_{i} \mu_{i}+q E_{\mathrm{F}}+E_{\text {correction }}
$$

where $E_{\mathrm{d}}$ is the energy of a supercell with the defect, $E_{\text {bulk }}$ is the energy of a corresponding defect free supercell, $n_{i}$ is the number of atoms removed or added in the defective supercell, and $\mu_{i}$ their chemical potential. For a defect of charge $q$, the chemical potential for electrons, i.e. the Fermi energy $E_{\mathrm{F}}$, has to be included as well. A final correction term can be added, e.g., to remove strain energies [40] or electrostatic interactions [41,42] due to periodic boundary conditions. It is good to stress at this point that the chemical potential of species $i$ is the energy of the reservoir that atoms are exchanged with. As an example, for a vacancy in an elemental solid such as bcc Fe, the chemical potential is of course the energy of bulk 
bcc Fe. If the system is, instead, a binary compound, the relevant reference state is not univocally defined, therefore in order to compare with experimental results one need to know what is the reservoir in the experiment itself. The formation energy defined in this way can be then used to estimate the equilibrium concentration of a defect in the system at a certain temperature in the dilute limit with an Arrhenius equation such as in Eq. 1.1.

Another important detail to take into account in the study of defects is structural relaxation. As it can be imagined, a defect in the lattice will lead to a local rearrangement of the surrounding atoms, and this is fundamental in the estimation of formation energies. Relaxations can be quite long ranged, therefore the size of the supercell plays an important role also on this effect. As an example, for $\mathrm{C}$ interstitials in FM bcc Fe, it is well known that a strong strain field is induced in the lattice, therefore large supercells and correction schemes [40] are needed in order to obtain accurate solubilities.

Regarding diffusion of defects, it is possible to calculate energy barriers within static DFT calculations and then employing transition state theory [43] in order to predict rates. The calculation of barriers is very often made with the previously mentioned NEB method [44], which enables to find the minimum energy path to go from the initial to the final state. In this method, several images of the system are created with the diffusing atom in different positions going from the initial to the final state. Each image of this atom is connected to the neighboring one with fictitious springs, in order to avoid the collapse of one image onto the other, and all atoms in the supercell in each replica are allowed to relax. In addition, the component of the spring force perpendicular to the path is projected out so that it does not influence the relaxation of the images perpendicular to the path. Several implementations [44, 45] of this method have been developed, as well as other methods that enable the calculation of minimum energy path or minimum free energy paths $[46,47]$.

\subsubsection{Random alloys}

The theoretical modeling of a random substitutional alloy requires great care in first principles calculations. The first way that could come to mind is to use several supercells with unbiased random number generated distributions of the different chemical species in the material, and then average the results over the different samples. However, this approach is in practice found to often require a very large statistics. Much cheaper methods are available. Here we need to distinguish between two types of approach: supercell methods and Green's function methods based on scattering theory. The latter include the CPA method previously mentioned, which consists in replacing the disordered lattice with an effective medium that reproduces the average scattering properties of the real atoms as embedded in the medium. This technique is much more elaborated than this easy explanation, but it has not been used in this work, therefore I redirect the interested reader to more detailed descriptions [48].

For what concerns supercell methods, the most efficient known approach to represent chemical disorder is the so-called special quasi-random structure (SQS) 
[49]. In this method, the atoms of the substitutional disordered alloy are placed in the lattice so that the correlation function between each type of atom mimic the average correlation function in an infinite lattice, at least for the shorter coordination shells. To be more clear, let us take a simple example. If we consider a random $\mathrm{A}_{0.5} \mathrm{~B}_{0.5}$ alloy, we can expect that the nearest neighbor to an $\mathrm{A}$ atom will be on average half $\mathrm{A}$ atoms and half $\mathrm{B}$ atoms; this consideration can be done for every coordination shell included in the calculation, so that the particular cell used will have the correlation function expected for an infinite lattice. Of course, this method is still affected by the size of the supercell, since all the important interactions between shells need to be included [50]; however, it turns out to be much more efficient then the random distribution of atoms in the lattice. With a generalization of this method, one can as well reproduce different degrees of short range order.

One advantage of the SQS method over the CPA approach is that in the former structural relaxations can be easily performed. In a real crystal we can expect that chemical disorder will induce local lattice relaxations, and this will affect the energetics of the system as much as in calculations involving defects. However, in the SQS approach modeling of defects cannot be performed with one single structure since the defect would experience only one particular local environment. In this context, a statistical average over several configurations need to be performed in order to improve the description.

Another method that has been developed in the past to study configurational disorder is th cluster-expansion method [51]. In this formalism, an alloy is considered as a collection of clusters, which can be univocally defined taking an orthonormal basis founded on discrete spin variables $\sigma_{i}$, defining the occupancy of a particular site $i$ in the cluster. With this method, any property of an alloy can be decomposed in the orthonormal basis, and once the relevant interactions are obtained, thermodynamical properties can be obtained with the use of a generalized Ising Hamiltonian. For a more detailed presentation of the cluster expansion method, the reader is referred to, e.g., Ref. [52].

\subsection{Microscopic description of magnetism}

As mentioned in Sec. 1.2, magnetism in solids is a collective electronic phenomenon. The exact way to describe this effect would be to take into account the many-body wavefunction of the electrons; this is of course highly unpractical, therefore methods to solve this problem in an easier way have been devised. DFT enables to calculate the ground state of magnetic materials, but in general it has to been complemented with other models to describe finite-temperature excitations.

A method that has the theoretical framework to describe correlation effects and electronic excitations in magnetic materials is dynamical field theory (DMFT) $[53,54]$. This theory is based on the Hubbard model, where the lattice problem is mapped onto an impurity model of an atom embedded in an electron bath. Even though very successful, it still has some drawbacks. Since it is based on a model Hamiltonian, its parameters have to be derived. Often they are just varied to 
match some experimentally known property, reducing the ab initio character of this method. Schemes to calculate the parameters fully from first principles are available [55, 56], even though rarely used [57]. Because of its mean-field nature, DMFT is not able to describe critical regions, since local fluctuations are not included. As an example, the Curie temperature of bcc Fe is heavily overestimated in DMFT [58]. For the same reason, short range order effects, which are still present above $\mathrm{T}_{C}$, cannot be captured either. In addition, local environment effects are also neglected due to the mapping on an impurity problem. Moreover, a definitive proof of the ability of DMFT to accurately calculate interatomic forces is still missing, therefore efficient schemes for performing structural relaxations or actual MD simulations cannot be carried out in this framework. However, the DMFT community is trying to improve methods in order to get there, even though generally based on energy calculations rather than force calculations [59, 60]. As a final remark, its computational cost is still often beyond feasibility for the description of systems with low symmetry or defects.

It is therefore important to keep developing DFT-based methods that enable an approximate description of all the degrees of freedom of the system, allowing also to describe critical regions. To date, many semi-classical models are routinely employed in order to study temperature effects and more general properties in magnetic systems. These methods are often based on the Heisenberg Hamiltonian defined in Eq. 1.3.

\subsubsection{Heisenberg Hamiltonian}

The semiclassical Heisenberg Hamiltonian (Eq. 1.3) describes a static lattice of spins or magnetic moments with a constant magnitude and well defined constant interaction $\left(J_{i j}\right)$. It can be shown that the classical Hamiltonian can be derived from its quantum version (where the moments are replaced by the spin operators $\hat{S}$ ) in the limit of $\hbar \rightarrow 0, s \rightarrow \infty$ (where $s$ is the spin quantum number). Requiring that $s \rightarrow \infty$ corresponds to requiring that the spin can point in any direction in space, going therefore from quantized to continuum regime. It can be seen that the exchange interactions $J_{i j}$ define the type of magnetic order of the system: if $J_{i j}>0$, parallel spins are favored, whereas if $J_{i j}<0$ antiparallel spins lower the energy of the system. Exchange interactions are related to the exchange integral that appears in the Schrödinger equation, and in a 2-electrons model they are given by the energy difference between the triplet and the singlet states (where exchange interaction and integral coincide). For solids, the relation is more complex, but several methods for the calculation of these quantities within DFT have been developed in the years [61-63].

Although the basic Heisenberg Hamiltonian has been proven successful for the prediction of $T_{C}$ of materials with very well localized moments, in general one needs to be careful. Examples of fully Heisenberg systems are quite rare, and often one needs to increase the flexibility of the Hamiltonian to be able to model accurately a real material. A first example is that in the basic Heisenberg model, the magnitude of the moments is considered fixed and constant over the whole lattice. However, this might not hold for a realistic system, so that one can make 
the exchange interactions $J_{i j}$ independent on the magnetic moments size:

$$
H=-\sum_{i \neq j} J_{i j} \mathbf{e}_{i} \cdot \mathbf{e}_{j}=-\sum_{i \neq j} \tilde{J}_{i j} \mathbf{m}_{i} \cdot \mathbf{m}_{j},
$$

with $\tilde{J}_{i j}=J_{i j} /\left(\left|\mathbf{m}_{i}\right|\left|\mathbf{m}_{j}\right|\right)$, and the sums run over both $i$ and $j$. It is important to notice here that $\tilde{J}_{i j}$ does not depend on the size of the moments $i$ and $j$, whereas $J_{i j}$ does.

Another assumption of the Heisenberg model is that the exchange interaction between two moments does not depend on the environment, so that independently if the system is in, e.g., a ferromagnetic or paramagnetic state, the exchange interaction between atom $i$ and $j$ is always the same. However, this is typically not true, especially if one tries to study ferromagnetic metals with this model. As an example, it has been shown in [62] that for bcc Fe (which is often considered a Heisenberg system) the exchange interactions dramatically change if calculated with a FM or a PM background. It has been also shown there that the $J_{i j} \mathrm{~s}$ calculated with the PM background give a very good estimate of $T_{C}$. This is due to the fact that, near $T_{C}$, the magnetic system resembles more a PM phase rather than a FM one, therefore the relevant exchange interactions are the ones calculated with a PM background. For thermal excitations at low temperature, instead, the relevant $J_{i j}$ s are the ones calculated with FM background. The effect of the environment is even more pronounced when one tries to apply the Heisenberg model to more itinerant systems. In these cases (e.g., in fcc Fe-based magnetic alloys), the exchange interactions can even change sign just due to the particular configuration of the surroundings.

An additional important detail is that the Heisenberg model is based on a static lattice. In reality, of course, atoms vibrate, therefore a distance dependence of the $J_{i j}$ s can as well be taken into account. This can lead to a smooth relation between the value of the exchange interaction and the distance between the moments as it is observed in $\mathrm{CrN}$ [63], but it can also lead to a very complex behavior for which taking into account the distance between the pair might not be enough $[64,65]$, as seen in bcc Fe.

Further extensions of the Heisenberg Hamiltonian can be applied in order to include anisotropy or spin-orbit effects [66], however these are not studied in the present work, therefore they will not be discussed.

As a final remark, I would like to stress that the Heisenberg Hamiltonian deals only with the transversal magnetic degrees of freedom. However, if one wants to apply it to systems with a certain degree of itineracy, this is not enough, and one needs to find a way to include fluctuations on the longitudinal degree of freedom as well.

\subsubsection{Longitudinal spin fluctuations}

Longitudinal spin fluctuations (LSF) theory is an active research topic [67-71]. LSF are related to electronic excitations in a more intimate way than magnetic transverse degrees of freedom, and they are typically faster than these last ones; nonetheless, semi-classical models that allow for the approximate inclusion of this 
effect in the framework of the Heisenberg Hamiltonian have been developed [68, 72]. A common way is to add to the Heisenberg Hamiltonian an on-site term which depends on the surrounding only in a mean-field manner:

$$
H_{\mathrm{LSF}}=-\sum_{i \neq j} \tilde{J}_{i j} \mathbf{m}_{i} \cdot \mathbf{m}_{j}+\sum_{i} E\left(m_{i}\right) .
$$

The last term in the Hamiltonian describes the dependence of the energy of moment $i$ on its own magnitude $m_{i}$. From Landau theory, this energy can be expanded in even powers of the moment size:

$$
E\left(m_{i}\right)=\sum_{n=0}^{\infty} a_{2 n} m_{i}^{2 n} .
$$

This expansion truncated to $4^{\text {th }}$ order already describes qualitatively the dependence of the on-site energy on the moment size. This model is valid only at high temperatures, above the transition temperature, because of its single-site nature. It has been used to explain properties of the ferromagnetic elemental solids (bcc Fe, Ni and Co)[68-71] and Heusler alloys [67], to name a few. The LSF energy shows very different behavior for itinerant and localized moments system in a paramagnetic environment, as can be seen in Fig. 2.3. In this regime, itinerant systems (dotted line in Fig. 2.3) show a minimum energy for null moment, whereas the localized moment system (solid line) has minimum energy at some finite value. This means that, at high temperatures, the moments of an itinerant system are non-zero only because of entropy. For an itinerant system, therefore, temperature will increase the average size of the moments. For a localized system, this is not as straightforward due to the asymmetry of the energy landscape.

A key quantity to calculate in this context is the magnetic entropy. An expression often used is a mean field approximation derived from basic quantum mechanical considerations and translated to the classical moments by taking the proper limits $\hbar \rightarrow 0, s \rightarrow \infty$, with $s$ being the total spin quantum number for an atom. In the quantum system, the entropy can be derived counting all the possible states that the spin can assume in the paramagnetic state; taking the limits, the entropy becomes $[16,71]$ :

$$
S^{\mathrm{mag}}=k_{B} \log (m+1)
$$

Another form of the entropy can be analytically derived assuming an Hamiltonian as in Eq. 2.30 with a quadratic on-site term, which is consistent with an itinerant ferromagnet. In this approximation, the entropy reads [69]:

$$
S^{\mathrm{mag}}=3 k_{B} \log m .
$$

This expression can be employed also for a more localized moment ferromagnet as bcc Fe if the minimum of the energy landscape is deep and can be approximated with a parabolic term, in a harmonic-approximation fashion. In general, the size of the moment $m$ at a certain temperature $T$ can then be calculated minimizing the free energy including either of these entropic terms. 


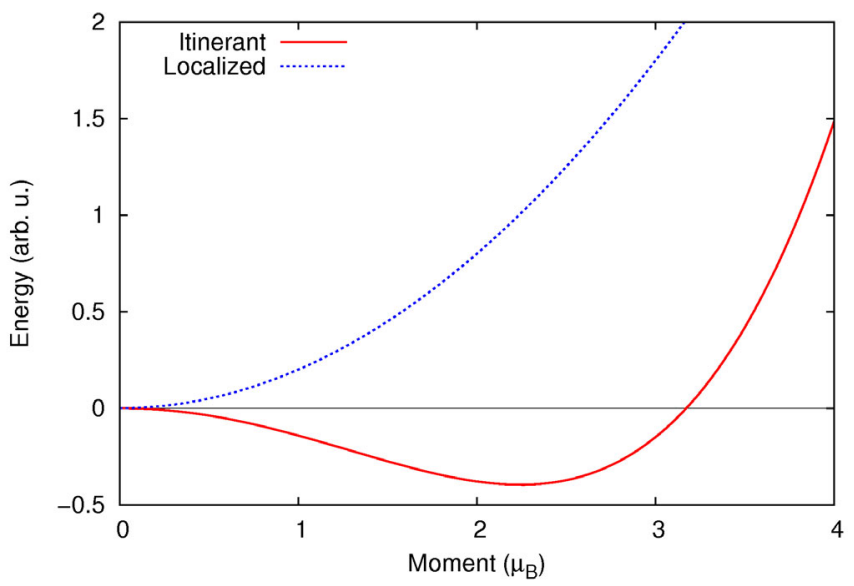

Figure 2.3: Typical on-site LSF energy as a function of the moment size for an itinerant (blue dotted line) and a localized moment (red solid line) systems in a paramagnetic background. The itinerant system has minimum energy for $m=0$, whereas the localized moment system has it for $m>0$.

The magnetic entropy in Eq. 2.33 is derived taking into account also another detail: the phase space measure (PSM) [73]. In order to introduce this concept, let us start from the partition function for a moment $i$ in a solid in a totally disordered state:

$$
\begin{aligned}
Z_{i} & =\int \mathrm{d} \mathbf{m}_{i} \exp \left(-\frac{-\sum_{j} \tilde{J}_{i j} \mathbf{m}_{i} \cdot \mathbf{m}_{j}+E\left(m_{i}\right)}{k_{B} T}\right) \\
& \approx \int \mathrm{d} \mathbf{m}_{i} \exp \left(-\frac{E\left(m_{i}\right)}{k_{B} T}\right),
\end{aligned}
$$

where the Heisenberg term has disappeared because $\left\langle\mathbf{m}_{i} \cdot \mathbf{m}_{j}\right\rangle \approx 0$ in the paramagnetic state. Here, it is useful to pass to spherical coordinates in order to separate the transversal and longitudinal degrees of freedom. However, at this point, the magnetic nature of the system needs to be taken into account. For a more localized moments system, the longitudinal degree of freedom can be thought to be completely decoupled from the transversal ones, therefore the problem becomes one-dimensional and the partition function is:

$$
Z_{i}=\int \mathrm{d} m_{i} 1 \cdot \exp \left(-\frac{E\left(m_{i}\right)}{k_{B} T}\right)
$$

where constants from the angular integration are neglected. If the system, instead, shows some degree of itineracy, the different degrees of freedom cannot be decoupled and the Jacobian determinant needs to be taken into account:

$$
Z_{i}=\int \mathrm{d} m_{i} m_{i}^{2} \exp \left(-\frac{E\left(m_{i}\right)}{k_{B} T}\right) .
$$


The terms 1 and $m_{i}^{2}$ are defined as the PSM, and they lead to different behaviors: the 3D PSM $\left(m_{i}^{2}\right)$ assigns more statistical weight to large moments, whereas the 1D (1) one treats at the same level moments with different size. It is not well defined which PSM one needs to use and in which cases [73, 74]; in this work, we employ the 3D PSM whenever we include the LSF term to fully account for the dimensionality of the problem.

\subsubsection{Disordered local moment approach}

So far, I have been describing the semi-classical models that can be employed in the study of magnetism, but I have not mentioned how these can be exploited in the DFT framework. With spin-polarized DFT, it is easy to represent ordered structures, that can be either collinear or noncollinear, and with also possible addition of spin-orbit coupling. However, it is not as straight-forward to model the PM phase. Of course, as should be clear from the previous discussions, modeling the PM phase as nonmagnetic leads to completely wrong physics since the atomic magnetic moments are usually still alive above the critical temperature. The disordered local moment (DLM) approach allows to represent the PM phase within this type of calculations.

The DLM approach is a semi-classical model based on the work of Hubbard and Hasegawa [75-79] and firstly implemented in the CPA framework by Gyorffy et al. [80]; later on, it has been developed also in supercell approaches [81]. Its applicability is motivated by the fact that most often the magnetization density can be viewed in the localized moment picture.

In this approach, the PM phase is modeled by distributing up and down magnetic moments on the atoms. In the CPA framework, this can be seen as an $\mathrm{A}_{0.5} \mathrm{~B}_{0.5}$ alloy, with $\mathrm{A}$ being spin-up moments and $\mathrm{B}$ spin-down. In the supercell approach, two methods have been developed: the first one is based on a magnetic SQS, in analogy with the alloy; the second one, known as magnetic sampling method (MSM), is based on the use of different configurations in which the moments are randomly distributed, and physical properties are then obtained as averages over all the configurations. It has been shown [81] that in the MSM method correlation functions and self-averaging properties converge to SQS and CPA results. The MSM method was used in this work also with noncollinear configurations of the magnetic moments, in order to have a better representation of the magnetic disorder.

In general, the DLM approach is an idealization of the PM phase formally representing temperature $T \rightarrow \infty$, or at least $J / T \rightarrow 0$, with $J$ being the strongest exchange interaction, for which the spin-correlation functions tend to zero. It is however possible to introduce different degrees of magnetic short range order in this approach, especially in the MSM implementation. It has been proven to be able to reproduce many interesting features due to the change in magnetic properties at high temperature [81-83], without the need to resort to expensive higher-level theories.

It has also been implemented together with MD simulations [84-87] (DLM$\mathrm{MD}$ ), where the configuration of the moments is changed every few timesteps. 
However, in this method the magnetic and vibrational degrees of freedom are not fully coupled, since the randomly changing DLM state represents an adiabatic fastmagnetism decoupling from the lattice. In order to include the interplay between lattice vibrations and magnetism, one needs to go beyond the full decoupling of magnetic and vibrational degrees of freedom.

\section{DLM relaxation}

What if one wants to study a magnetic system in the PM phase in presence of defects? Does the magnetic state influence the properties of defects? As shown in Ref. [82] for bcc Fe, the interatomic force constants soften quite importantly when going from the FM to the PM phase, therefore one can in general expect an effect of different magnetic states on the properties of defects.

This problem has been tackled in many different ways in the literature: a first way has been to employ FM-relaxed atomic positions and then perform MSM calculations on these positions [88]; another way has been to allow partial relaxation of the positions employing different MSM configurations, averaging results obtained from these different geometries [89]; another final one, was to perform DLM-MD simulations on defective supercells, so that in this way also the effect of vibrations is taken into account $[83,86]$. Another method based on spin-wave (SW) calculations has also been developed [90, 91] for the representation of the PM phase, enabling also structural relaxations, but its description is beyond the scope of this thesis. In general, it would be good, from a computational perspective, to have one single configuration of the atoms relaxed with the relevant magnetic state, on which one can perform static calculations.

For this reason, in paper II we have developed a method to perform structural relaxations in the PM phase based on the DLM approach (DLM relaxation). In this method, the structural relaxation is started taking into account some initial positions of the atoms (e.g., ideal lattice positions with the defect or pre-relaxed in the FM state) in a supercell containing the defect and drawing a random MSM configuration. Forces are calculated and symmetrized according to the symmetry of the underlying lattice in a spin-space average (SSA) fashion [82], and the atoms are moved according to this symmetrized forces. This procedure is repeated, changing at every step the MSM configuration, until the atoms reach a regime in which they fluctuate around some mean value. The final equilibrium positions are then obtained averaging over this steady-displacement regime.

The symmetrization of forces is an interesting detail to discuss: in experiments, a material in the high-temperature PM phase will show a symmetry because of time-average of the measurement; however, instantaneously, the atoms are not in equilibrium positions. Therefore, the static lattice approximation is not really describing the system (although quite successful). In addition, we have noticed that the results of relaxations with and without imposition of symmetry do not show relevant differences. This is due to the fact that the MSM configuration is changed at every step, acting on average according to the underlying symmetry.

Results from this method, which will be discussed in Sec. 3.2, show differences with, e.g., DLM calculations on FM-relaxed positions, and are in very good 
agreement with higher-level theory results [60], when such exist.

\subsubsection{Coupled atomistic spin dynamics - ab initio molecular dynamics}

What is left to discuss is how the moments evolve in time. Spin dynamics simulations are possible on a semi-classical level through the use of the Heisenberg Hamiltonian and equations of motions for the spin. The Landau-Lifshitz-Gilbert equations can be used in this context:

$$
\frac{\partial \hat{\mathbf{S}}_{i}}{\partial t}=-\frac{\gamma}{1+\alpha^{2}} \hat{\mathbf{S}}_{i} \times\left[\mathbf{H}_{\mathrm{eff}}+\mathbf{f}_{i}\right]-\gamma \frac{\alpha}{1+\alpha^{2}} \hat{\mathbf{S}}_{i} \times\left\{\hat{\mathbf{S}}_{i} \times\left[\mathbf{H}_{\mathrm{eff}}+\mathbf{f}_{i}\right]\right\}
$$

where $\gamma$ and $\alpha$ are the electron gyromagnetic ratio and the phenomenological damping factor, respectively, whereas $\mathbf{f}_{i}$ is the random force employed in Langevin dynamics to enforce the effect of temperature on the system (Sec. 2.2.2). $\mathbf{H}_{\text {eff }}$, instead, is the effective magnetic field experienced by moment $i$ due to all the other moments, and it is expressed as:

$$
\mathbf{H}_{\mathrm{eff}}=-\frac{1}{m_{i}} \frac{\partial H}{\partial \hat{\mathbf{S}}_{i}} .
$$

The size of the moments $m_{i}$ in this equation enters in the $\mathbf{H}_{\text {eff }}$ term and implicitly in the Hamiltonian $H$ through the exchange interactions $J_{i j}$, which depend on $m_{i}$ and $m_{j}$ as explained in Sec. 2.4.1 with Eq. 2.29. For the rest only the direction of the vectors is needed $\left(\hat{\mathbf{S}}_{i}\right)$.

These equations have been used in atomistic spin dynamics (ASD) simulations in order to predict and explain magnetic properties of materials [66], and also in conjunction with model interatomic potentials for the representation of lattice degrees of freedom in what is known as spin-lattice dynamics. However, it is highly desirable to employ the most accurate tools in simulations, and as already mentioned classical force fields suffer of serious problems with accuracy and reliability, whereas AIMD is in general much more reliable. In Ref. [92], a coupled ASDAIMD method has been developed and it has shown that the adiabatic interplay between magnetic and vibrational degrees of freedom is indeed playing a role in the dynamics of the system $\mathrm{CrN}$.

In this method, the spin and the lattice systems are developed in parallel and are able to intercommunicate. A regular simulation starts with a pre-equilibrated configuration of the atoms (e.g., by means of DLM-MD). Then, the distancedependent exchange interactions are assigned for each pair of atoms according to their distance and fed into a spin Monte Carlo simulation in order to get an equilibrated configuration of the magnetic moments. With this magnetic configuration, an AIMD step is performed, and from the new positions the exchange interactions are feeded into the ASD code in order to perform a spin-dynamics simulation of the same duration of the timestep used in AIMD. Then the new magnetic configuration is used for the next AIMD step, and this procedure is reiterated for the whole duration of the simulation. Here, one thing that has to be checked is the 
typical revolution time of the magnetic moments: the faster is the spin dynamics, the smaller will have to be the AIMD timestep in order to have reasonable magnetic forces acting on atoms at each step of the simulation. Anyway, in general a much smaller timestep has to be used in the spin dynamics part of the simulation (e.g., in bcc Fe we use $10^{-2}$ fs for ASD compared to 1 fs in the AIMD run) because it leads to better numerical convergence of spin trajectories; this is not a computational problem since ASD simulations are much cheaper than AIMD ones. 


\section{CHAPTER 3}

All DFT results in this chapter have been obtained with the Vienna ab initio simulation package (VASP)[93, 94] with the PAW method [22, 95]. Calculations concerning TiN have been carried out with the Armiento-Mattson exchange and correlation functional (AM05) [96]; for Fe, the PBE [24] exchange and correlation functional was employed, whereas calculations of $\mathrm{CrN}$ properties have been performed with $\mathrm{LDA}+\mathrm{U}$, in the formulation of Dudarev [97], with an effective value $\mathrm{U}^{\mathrm{eff}}=3 \mathrm{eV}$. For more computational details, the reader is referred to the papers and, for unpublished results, to the relative section.

\subsection{Ti vacancy diffusion in $\mathrm{TiN}$}

TiN is the prototype of transition metal nitrides (TMNs). These materials, made of a group IIIB-VIB metal and nitrogen, are refractory ceramics commonly employed as protective coatings on cutting tools [98] and as diffusion barriers in electronic devices [99] thanks to their extreme hardness [100], toughness [101], thermal stability [102], and good electrical conductivity [103]. In particular, they are employed as diffusion barriers because of the low diffusion rates of impurities in TMNs thin films.

$\mathrm{N}$ vacancies are the most common point defect in these materials. Experimental studies of $\mathrm{N}$ diffusivity in TiN are available in the literature [104], although scattered over several orders of magnitude. Ti vacancies are assumed to be less important, nonetheless there are indications that metal vacancies might govern the spinodal decomposition of $(\mathrm{Ti}, \mathrm{Al}) \mathrm{N}$ pseudo-binary alloys $[105,106]$. Precise experimental information on this defect is lacking in the literature, therefore computational investigations can shine light on phenomena involving metal vacancies.

TiN, as most of the transition metal nitrides, has a B1 rock-salt structure 
from $0 \mathrm{~K}$ up to its melting point $T_{m} \approx 3250 \mathrm{~K}$ [107] and over a wide range of stoichiometries [108]. N defects in this system have been studied in the past years [109], finding $\mathrm{N}$ vacancy and $\mathrm{N}$ interstitial formation energies to be 2.41 and 4.60 $\mathrm{eV}$, respectively. In our calculations, we obtain a $\mathrm{Ti}$ vacancy formation energy of $2.86 \mathrm{eV}$, higher than for the $\mathrm{N}$ vacancy as expected, but lower than for the $\mathrm{N}$ interstitial.

The aim of this study (paper I) is to investigate diffusion rates of Ti vacancies in TiN by means of CD-NEMD, described in Sec. 2.1. For this, we started with NEB calculations for vacancy mediated diffusion of $\mathrm{Ti}$ atoms with the 0 $\mathrm{K}$ lattice parameter to have an indication of the maximum force field that can be employed in the NEMD calculations. The most plausible jump is between neighboring sites in the metal sublattice, i.e. along a $\langle 110\rangle$ direction. The resulting energy landscape, shown in the inset in Fig. 3.1, gives an energy barrier for diffusion of $4.26 \mathrm{eV}$. This entails that a maximum force field of intensity $F_{\max }=$ $3.3 \mathrm{eV} / \AA$, with a $<100>$ component of $2.2 \mathrm{eV} / \AA$, can be applied to the system without incurring instabilities. We therefore employ five force field intensities, with $<100>$ component ranging from 1.4 to $2.2 \mathrm{eV} / \AA$ in steps of 0.2 . We study diffusion at temperatures of 2200, 2400, 2600, 2800, and $3000 \mathrm{~K}$ employing the lattice parameter at that temperature by expanding the $0 \mathrm{~K} \mathrm{DFT}$ value with the experimental linear thermal expansion coefficient $\alpha \approx 9 \times 10^{-6} \mathrm{~K}^{-1}$.

At each temperature and force field, between 15 and 20 NEMD simulations are carried out, starting from independent atomic configurations pre-equilibrated at the temperature of interest for about $3 \mathrm{ps}$. The simulations are stopped either if a jump occurs, or when a jump is observed in at least $75 \%$ of the runs. These occurrence times are then fitted with a censored exponential distribution [36, 110], which allows to take into account in the fitting also the simulations where the jump has not occurred. The resulting nonequilibrium jump rates as a function of the force field intensity are shown in Fig. 3.1 with errorbars derived from the fitting. These values are then interpolated with Eq. 2.25, where $x_{T S 0}(T)$ is set to half of the distance between nearest-neighbors in the metal sublattice.

As it can be seen from Fig. 3.1, the nonequilibrium jump rates at high force field intensities are similar to each other, regardless of the temperature. However, decreasing the intensity, the rates start following the expected trend so that the equilibrium values extrapolated at zero force field show decreasing rate for decreasing temperature.

The equilibrium jump rates are plotted in Fig. 3.2 as a function of temperature. Ti vacancy jump rates are well represented by Arrhenius equation for diffusion (red line), where the extrapolated activation energy is $3.77 \pm 0.64 \mathrm{eV}$ (with uncertainty corresponding to two standard deviations), lower than the $0 \mathrm{~K}$ value of $4.26 \mathrm{eV}$. As a comparison, $\mathrm{N}$ vacancy diffusion jump rates taken from Ref. [111] are also shown (blue line), where the extrapolated activation energy is $3.65 \mathrm{eV}$. Therefore, the main difference in diffusion rates of $\mathrm{N}$ and $\mathrm{Ti}$ vacancies is due to the jump attempt frequency rather than the activation energy. These results are also compatible with experimental results for Ti vacancy diffusion obtained in $\mathrm{TiN} / \mathrm{NbN}$ superlattices across interfaces [112].

Further NEB calculations with expanded lattice parameter at 2200 and $3000 \mathrm{~K}$ 


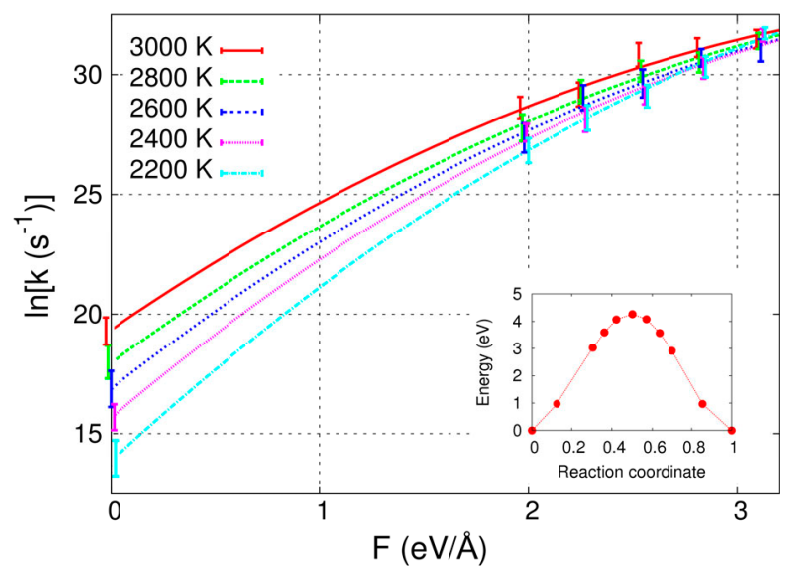

Figure 3.1: Nonequilibrium jump rates for Ti vacancy diffusion in TiN as a function of force field intensity $\mathrm{F}$ at temperatures of 2200, 2400, 2600, 2800, and $3000 \mathrm{~K}$. The inset shows the energy profile for diffusion along the [110] direction calculated with the NEB method at $0 \mathrm{~K}$. The line connecting the points is a guide for the eyes. Figure adapted with permission from D. Gambino, D. G. Sangiovanni, B. Alling, and I. A. Abrikosov, Physical Review B 96, 104306 (2017). Copyright 2017 by the American Physical Society.

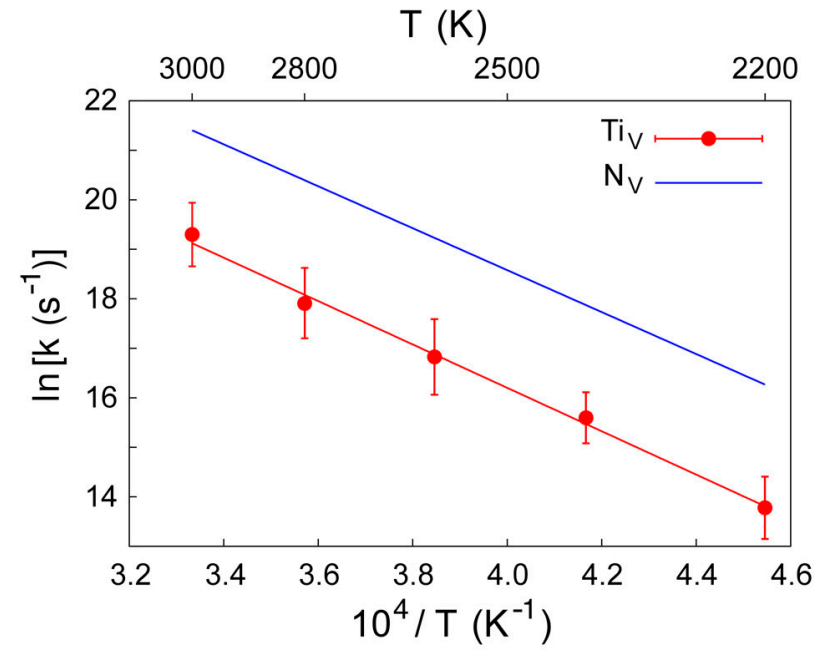

Figure 3.2: Logarithm of the equilibrium jump rates for Ti vacancy diffusion in TiN as a function of inverse temperature, showing Arrhenius behavior. As a comparison, $\mathrm{N}$ vacancy jump rate from Ref. [111] is also shown (blue line). Reprinted figure with permission from D. Gambino, D. G. Sangiovanni, B. Alling, and I. A. Abrikosov, Physical Review B 96, 104306 (2017). Copyright 2017 by the American Physical Society. 
give an energy barrier of 3.67 and $3.50 \mathrm{eV}$, respectively; therefore, the reduction in activation energy can be mainly attributed to thermal expansion of the lattice. The difference between these two values and the one extrapolated with Arrhenius fitting might be due just to statistical reasons, but we cannot rule out that anharmonic lattice vibrations alter the activation energy at high temperature.

\subsection{Lattice relaxation in magnetic materials in the high temperature paramagnetic phase}

In paper II, we have developed and tested a method to perform lattice relaxation of magnetic materials in the high temperature PM phase from first principles based on the DLM model. The method has been already discussed in Sec. 2.4.3, and in the following I am going to summarize the results obtained in that work. In addition, further yet not published results on defects in $\mathrm{PM} \mathrm{CrN}$ are going to be discussed.

The workflow of these calculations is the following: the DLM-relaxation is first performed for the system of interest; from here, a fixed configuration of atomic positions is obtained, on which static MSM calculations of the value of interest are carried out. The final result is the average over all these employed configurations at the fixed geometry.

\subsubsection{Fe-based materials}

Any method aimed at improving the description of magnetism in first principles calculations is often tested first on Fe because of its great technological importance and the interesting physics related to magnetic and structural transitions. We have chosen to test our DLM-relaxation method investigating a few very important cases: the formation energy of a single vacancy in bcc Fe, for which the literature is full of theoretical and experimental results; a $\mathrm{C}$ interstitial in bcc Fe, due to its technological importance in steels; finally, $\mathrm{Fe}_{1-x} \mathrm{Cr}_{x}$ alloys (with $\mathrm{x}=0.25,0.50$, and 0.75 ), where $\mathrm{Cr}$ is a very common alloying component to give stainless steels.

In table 3.1, the present results for the formation energy of a vacancy and a $\mathrm{C}$ interstitial in FM and PM bcc Fe are compared with theoretical $[60,88,91]$ and experimental [113-117] results from the literature.

For what concerns the vacancy, the change from FM to PM state affects considerably the formation energy, leading to a decrease of $0.50 \mathrm{eV}$. Performing a relaxation in the DLM state gives a further reduction of $0.09 \mathrm{eV}$, a non-negligible correction to the formation energy. Our results agree with recent DFT $+\mathrm{DMFT}$ calculations by Delange et al. [60], where the small difference may be attributed to employment of different lattice parameters and different representation of the PM state (our DLM state is a good representation of the PM phase in absence of magnetic short range order, whereas DMFT calculations in [60] were done at 1162 $\mathrm{K})$.

Regarding the DLM calculations by Sandberg et al. [88], which were performed on FM-relaxed positions, we argue that the accidental similarity with the present 


\subsection{Lattice relaxation in magnetic materials in the high temperature paramagnetic phase

Table 3.1: Vacancy and C interstitial formation energy for FM and PM bcc Fe. PM bcc Fe is modeled in the present work with the DLM approach, and the formation energy is calculated on FM- and DLM-relaxed positions. Other theoretical values from literature are calculated with DFT + DMFT [60], collinear DLM on FM-relaxed positions [88], and with the spin-wave (SW) method. The experimental values here reported are an average of several experimental results [113-117]. For $\mathrm{C}$ interstitial, we are not aware of any calculation in the PM phase. All values are in $\mathrm{eV}$.

\begin{tabular}{|c|c|c|c|}
\hline & \multirow{2}{*}{$\frac{\text { FM }(\mathrm{eV})}{\text { FM-relaxed }}$} & \multicolumn{2}{|c|}{$\mathrm{PM}(\mathrm{eV})$} \\
\hline & & FM-relaxed & $\overline{\text { DLM-relaxed }}$ \\
\hline \multicolumn{4}{|l|}{ Vacancy } \\
\hline This work & 2.20 & $1.70 \pm 0.06$ & $1.61 \pm 0.06$ \\
\hline DFT+DMFT [60] & 2.45 & $1.66 \pm 0.15$ & $1.56 \pm 0.13$ \\
\hline DLM [88] & 2.15 & $1.54 \pm 0.16$ & \\
\hline $\mathrm{SW}[91]$ & 2.13 & \multicolumn{2}{|c|}{1.98} \\
\hline Experimental [113-117] & 1.8 & \multicolumn{2}{|c|}{1.6} \\
\hline $\mathrm{C}$ interstitial & & & \\
\hline This work, octahedral site & 0.68 & $0.59 \pm 0.07$ & $0.41 \pm 0.06$ \\
\hline This work, tetrahedral site & 1.55 & $1.04 \pm 0.07$ & $0.62 \pm 0.06$ \\
\hline
\end{tabular}

results is due to the fact that in [88] some DLM configurations were discarded because of spin-flips. For the case of the vacancy, we did not observe this problem because constrained noncollinear calculations were employed, so that no configuration had to be discarded.

The SW results of vacancy formation energy [91] are larger than all the others for the PM phase, even though relaxation was included in that work. The large difference might be due to the fact that only planar ordered configurations of the moments are employed in the SW method.

Our result is in perfect agreement with the average of several experimental results [113-117]; the exact agreement, however, is only accidental since in this work we have not taken into account any contribution from, e.g., vibrations and magnetic short range order.

Regarding the results for the $\mathrm{C}$ interstitial, here the formation energy $E_{C}^{f}$ is defined as:

$$
E_{\mathrm{C}}^{f}=E_{\mathrm{C}-N \mathrm{Fe}}-N E_{0}(\mathrm{Fe})-E_{0}(\mathrm{C}),
$$

where $E_{\mathrm{C}-N \mathrm{Fe}}$ is the energy of a supercell with $N$ Fe atoms and one $\mathrm{C}$ atom in either octahedral or tetrahedral site, $E_{0}(\mathrm{Fe})$ is the energy of one Fe atom in the relevant magnetic state (FM or DLM), and $E_{0}(\mathrm{C})$ is the energy of one $\mathrm{C}$ atom in the diamond structure. Here we choose to use $\mathrm{C}$ in diamond as reference state rather than graphite, which is the true ground state, for practical reasons related to van der Waals interactions $[118,119]$. In addition, the exact absolute value of 


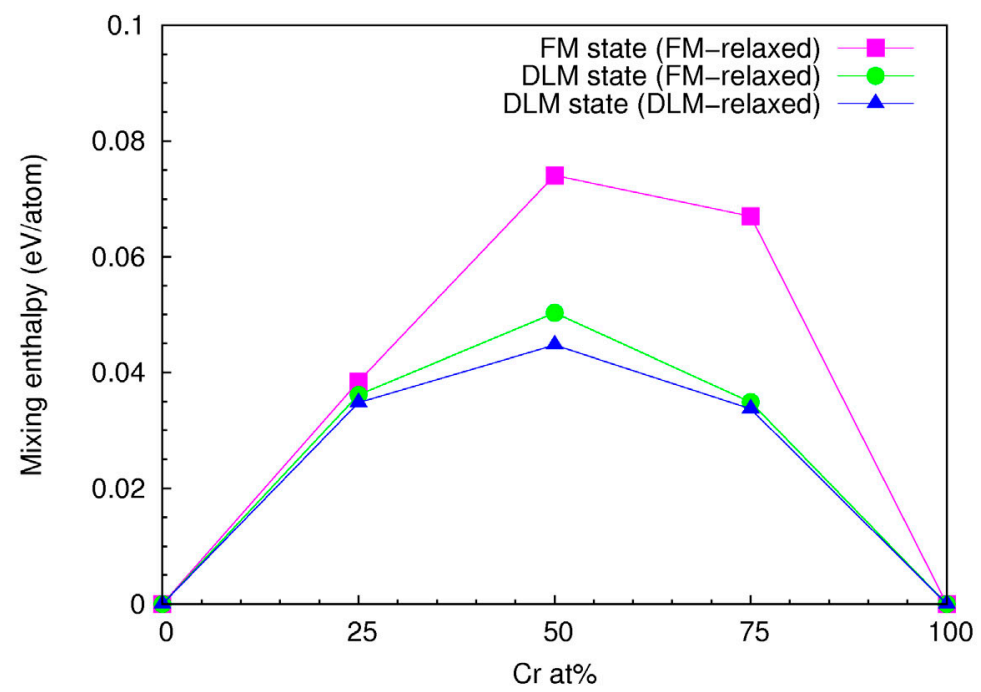

Figure 3.3: Mixing enthalpy of bcc $\mathrm{Fe}_{1-x} \mathrm{Cr}_{x}$ alloys in FM state (purple), DLM state on FM-relaxed positions (green), and DLM state on DLM-relaxed positions (blue) for compositions $x=0.25,0.50,0.75$. Reference states are FM and DLM bcc Fe (depending on the relevant magnetic state), and NM bcc Cr. Reprinted figure with permission from D. Gambino and B. Alling, Physical Review B 98, 064105 (2018). Copyright 2018 by the American Physical Society.

the formation energy is, for our purpose here, of less importance than differences between different models. Finally, in steels the relevant C-rich phase is most often cementite $\left(\mathrm{Fe}_{3} \mathrm{C}\right)$. The results for the $\mathrm{C}$ atom in tetrahedral position were not included in paper II. What is surprising in these results is that the reduction in formation energies due to the bare change in magnetic state is smaller than the reduction due to the DLM-relaxation for both octahedral and tetrahedral sites. It is also interesting to notice that the energy difference between the octahedral and tetrahedral sites becomes small. The octahedral site is known to be the stable position for $\mathrm{C}$ atoms in bcc $\mathrm{Fe}$, and the tetrahedral site the transition state for jumps between octahedral sites; such a reduction between the energy of these two sites might have important repercussions on relative stabilities and diffusion rates, therefore they should be investigated further.

These results might be affected by some computational problems, since we found that it is sometimes difficult to converge calculations with interstitials. However, we expect only small differences in the formation energies. Another detail to take into account is that $\mathrm{C}$ interstitials induce strong elastic strains in bcc Fe and, as already mentioned in Sec. 2.3.1, convergence with supercell size and removal of strain energies should be undertaken in order to have a quantitative analysis of these effects.

Last but not least, we have investigated the effect of relaxation in the DLM state on the mixing enthalpy of bcc $\mathrm{Fe}_{1-x} \mathrm{Cr}_{x}$ random alloys for compositions 
$x=0.25,0.50$, and 0.75 , defined as:

$$
\Delta H_{\text {mix }}\left(\mathrm{Fe}_{1-x} \mathrm{Cr}_{x}\right)=E\left(\mathrm{Fe}_{1-x} \mathrm{Cr}_{x}\right)-(1-x) E(\mathrm{Fe})-x E(\mathrm{Cr}) .
$$

In this equation, the reference state for Fe depends on the relevant magnetic state, whereas for $\mathrm{Cr}$ the reference state is always nonmagnetic bcc $\mathrm{Cr}$. The chemical disorder is modeled here with SQS structures [49]. As already known from previous investigations $[120,121]$, bcc $\mathrm{Fe}_{1-x} \mathrm{Cr}_{x}$ alloys show an anomalous small solubility range for low $\mathrm{Cr}$ concentrations in the FM phase with negative mixing enthalpies; however, when going to the PM phase, mixing enthalpy is positive for all concentrations, but with a reduction in magnitude for intermediate values. In Fig. 3.3, the formation enthalpy for the previously mentioned concentrations is shown for the FM state (purple), DLM state on FM-relaxed positions (green), and DLM state on DLM-relaxed positions (blue). The miscibility anomaly in the FM mixing enthalpy cannot be seen because it is confined to small concentrations of $\mathrm{Cr}$ in $\mathrm{Fe}$, however its presence can be guessed by the asymmetry of the mixing enthalpy profile. We observe a reduction in the mixing enthalpy due to the DLM relaxation of $\sim 10 \%$ for the 50-50 composition, whereas for the other two compositions the reduction is lower $(\sim 3 \%)$. This effect is smaller for $x=0.25,0.75$ because the system is closer to the pure elements, therefore more similar to a pure ideal lattice where lattice relaxations do not take place.

\subsubsection{Defects in paramagnetic $\mathrm{B} 1 \mathrm{CrN}$}

$\mathrm{CrN}$ is part of the transition metal nitrides previously described in Sec. 3.1. However, in contrast to the other components of the group, it is a semiconductor [122] and shows magnetic properties [123] that play an important role in a low temperature phase transition from an orthorombic to the usual rock-salt structure typical of this class of materials. Many theoretical investigations on this material have been undertaken in the past to explain the structural transition [5, 81, 124], the effect of magnetism, vibrations and their interplay [84, 85, 92], as well as some defects properties [86].

In Ref. [86], Mozafari et al. investigated the stability of different $\mathrm{N}$ defects in CrN by means of MSM calculations (on FM-relaxed positions) and DLM-MD simulations, as well as the impact of $\mathrm{N}$ vacancies and $\mathrm{N}$ interstitials in the form of dumbbells (see Fig. 3.4) on the density of states (DOS).

In this work, we have investigated defects that could lead to $\mathrm{N}$ overstoichiometry in this material, i.e. Cr vacancies, $\mathrm{N}$ dumbbells, their combination in few different configurations (Fig. 3.5), and $\mathrm{N}$ antisites ( $\mathrm{N}$ in a $\mathrm{Cr}$ site). In Fig. 3.4 and 3.5 only the conventional B1 cell with the defect is shown for clarity. We have performed calculations on a $3 \times 3 \times 3$ supercell, consisting of 216 lattice sites, which correspond to a stoichiometry $\mathrm{CrN}_{1.01}-\mathrm{CrN}_{1.02}$. For the single defects $(\mathrm{Cr}$ vacancy and $\mathrm{N}$ dumbbell), we have performed also calculations with a $2 \times 2 \times 2$ supercell ( 64 lattice sites), for an overstoichiometry of $\sim 0.03$. These stoichiometries have been observed in experiments [126], therefore they are relevant to investigate from first principles to understand the effect of more or less concentrated defects on the properties of this material. The cutoff energy for plane-waves expansion employed 


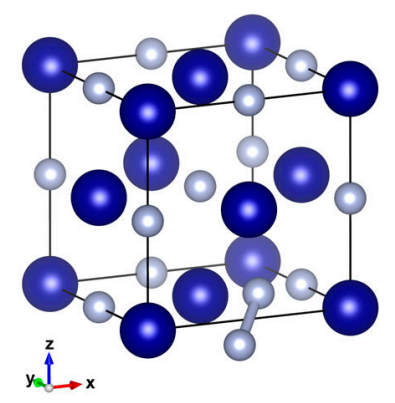

Figure 3.4: The lowest energy configuration of a N-N dumbbell in B1 CrN, directed along the [111] direction. Only the conventional cell is displayed for clarity purposes. N atoms are represented by small grey balls, $\mathrm{Cr}$ atoms by large blue balls. The structural representation is made with VESTA [125].

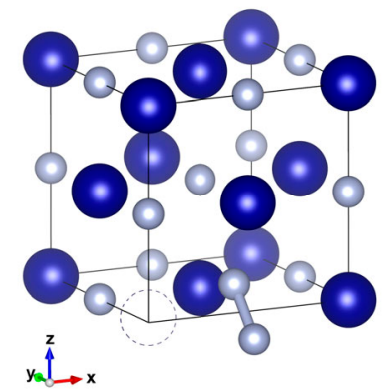

(a)

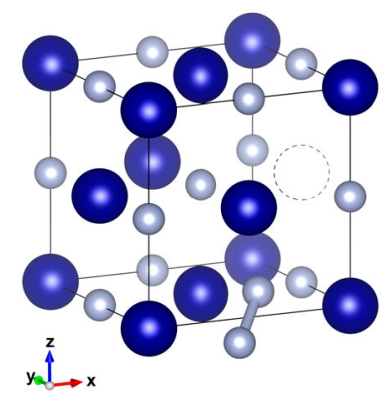

(c)

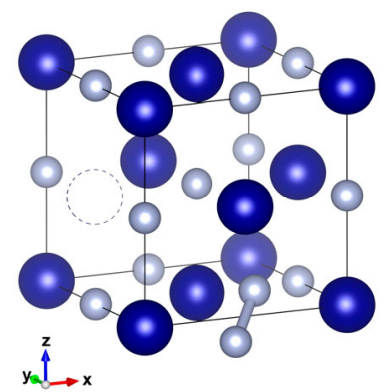

(b)

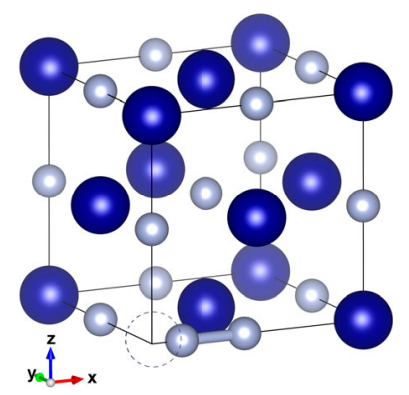

(d)

Figure 3.5: Combined Cr vacancy and $\mathrm{N}$ dumbbell in different configurations in a conventional B1 cell. The $\mathrm{Cr}$ vacancy is highlighted with a dashed empty circle. The $\mathrm{N}$ dumbbell is oriented in the [011] direction in (a), the [111] direction in (b) and (c), and in the [100] direction in (d). All the atoms other than the $\mathrm{N}$ in the dumbbell have been left in ideal lattice positions for clarity purposes. The structural representations are made with VESTA [125]. 
Table 3.2: Formation energies $\left(E^{f}\right)$ of the Cr vacancy $\left(\mathrm{V}_{\mathrm{Cr}}\right), \mathrm{N}$ dumbbell $\left(\mathrm{D}_{\mathrm{N}}\right), \mathrm{N}$ antisite $\left(\mathrm{A}_{\mathrm{N}}\right)$, and combined $\mathrm{Cr}$ vacancy and $\mathrm{N}$ dumbbell $\left(\mathrm{D}_{\mathrm{N}}[\mathrm{xyz}]+\mathrm{V}_{\mathrm{Cr}}\right)$ in DLM $\mathrm{CrN}$, with [xyz] indicating the orientation of the $\mathrm{N}$ dumbbell. The interaction energy $\left(E^{i}\right)$ between defects is also reported. Labels (a), (b), (c), and (d) for the combined defects refer to Fig. 3.5. All values are in $\mathrm{eV}$.

\begin{tabular}{ccccc}
\hline \hline & \multicolumn{2}{c}{$E^{f}(\mathrm{eV})$} & & $\mathrm{E}^{i}(\mathrm{eV})$ \\
\cline { 2 - 3 } & $2 \times 2 \times 2 \mathrm{sc}$ & $3 \times 3 \times 3 \mathrm{sc}$ & & $3 \times 3 \times 3 \mathrm{sc}$ \\
\hline $\mathrm{V}_{\mathrm{Cr}}$ & 2.44 & 2.48 & - \\
$\mathrm{D}_{\mathrm{N}}[111]$ & 3.81 & 3.61 & - \\
$\mathrm{A}_{\mathrm{N}}$ & & 6.91 & - \\
$\mathrm{D}_{\mathrm{N}}[011]+\mathrm{V}_{\mathrm{Cr}}(\mathrm{a})$ & & 4.75 & \\
$\mathrm{D}_{\mathrm{N}}[111]+\mathrm{V}_{\mathrm{Cr}}(\mathrm{b})$ & & 6.07 & \\
$\mathrm{D}_{\mathrm{N}}[111]+\mathrm{V}_{\mathrm{Cr}}(\mathrm{c})$ & & 5.88 & \\
$\mathrm{D}_{\mathrm{N}}[100]+\mathrm{V}_{\mathrm{Cr}}(\mathrm{d})$ & & 5.23 & -0.02 \\
\hline \hline
\end{tabular}

in DFT calculations is $400 \mathrm{eV}$ while sampling the Brillouin zone on $3 \times 3 \times 3 \mathrm{k}$-points mesh. The structures have been relaxed with the DLM-relaxation method. We use collinear magnetic moments for computational reasons, having also in mind from paper II that the representation of the DLM state with collinear or noncollinear moments has very similar energy. For the MSM calculations, we employ 25 magnetic configurations, which is enough in this system to obtain converged average energies within $1 \mathrm{meV}$ /formula unit.

Defect formation energies calculated with equation 2.28 are shown in table 3.2 , together with the interaction energies between defects defined as:

$$
E^{i}\left(D_{N}+V_{C r}\right)=E\left(D_{N}+V_{C r}\right)-E\left(D_{N}\right)-E\left(V_{C r}\right),
$$

where $E\left(D_{N}+V_{C r}\right), E\left(D_{N}\right)$, and $E\left(V_{C r}\right)$ are the energies of a supercell with the combined $\mathrm{Cr}$ vacancy-N dumbbell defect, a supercell with a $\mathrm{N}$ dumbbell, and a supercell with a $\mathrm{Cr}$ vacancy, respectively. Negative values of the interaction energy indicate that the combined defect is more stable than the isolated ones.

For the single defects, the $\mathrm{Cr}$ vacancy results to be the defect with the lowest formation energy, followed by the $\mathrm{N}$ dumbbell. The $\mathrm{N}$ antisite has a very large formation energy, therefore it is going to be disregarded in the rest of the discussion. There is also an effect due to the supercell size, for which the formation energy of the Cr vacancy is slightly increased, and for the $\mathrm{N}$ dumbbell is considerably decreased. Speaking of the interaction energy between defects, we observe that in every case the combined defect is energetically favored compared to the isolated defects, probably due to the larger space available for the dumbbell. The combination of defects with the strongest interaction has the $\mathrm{N}$ dumbbell oriented in the [011] direction, and the $\mathrm{Cr}$ vacancy in a first nearest neighbor site. It is also interesting to notice that the relaxation of this structure (Fig. 3.5a) started with the dumbbell in [111] direction as the other ones, but then the orientation 


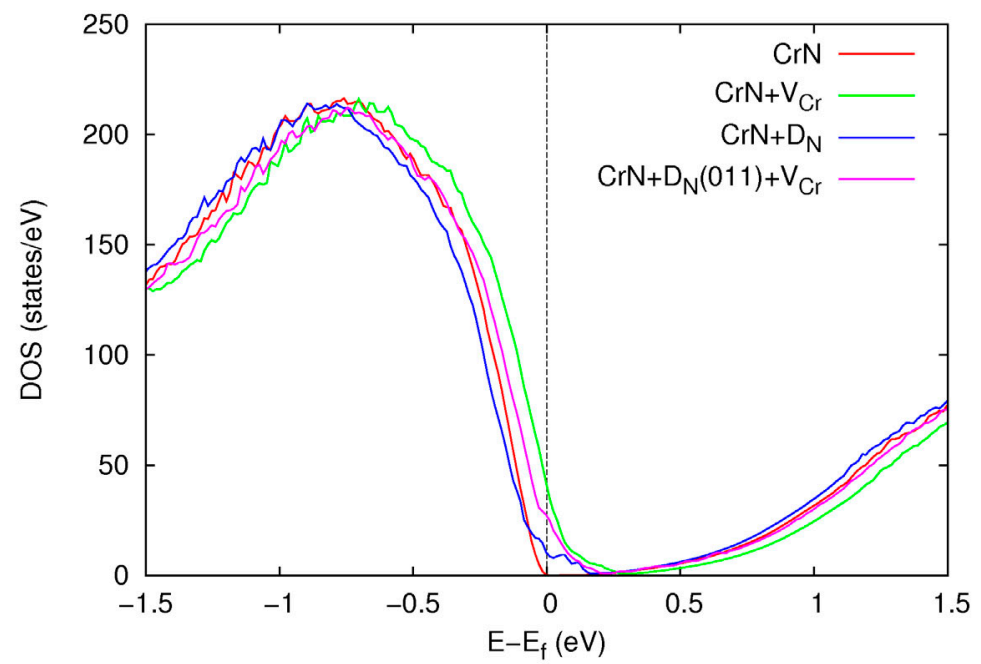

Figure 3.6: Electronic DOS in the vicinity of the Fermi level for defect free $\mathrm{CrN}$ (red), $\mathrm{CrN}$ with a $\mathrm{Cr}$ vacancy (green), $\mathrm{CrN}$ with a $\mathrm{N}$ dumbbell (blue), and with the combined $\mathrm{Cr}$ vacancy- $\mathrm{N}$ dumbbell in [011] direction (purple). The zero is taken as the Fermi energy.

changed during the relaxation giving a more stable structure in [011] orientation.

In Ref. [86] it was argued that $\mathrm{N}$ dumbbells induce a n-type bandstructure in CrN. A simple model to explain this is based on the fact that, for a $\mathrm{N}$ vacancy, three electrons are released by the bonds with neighboring $\mathrm{Cr}$ atoms, which will go in the conduction band (giving n-type semiconductor); if one thinks now to put a $\mathrm{N}$ dimer in place of the $\mathrm{N}$ vacancy with a double bond rather than triple (as suggested by the N-N distance in the dumbbell, larger than in a $\mathrm{N}_{2}$ molecule), this will catch two electrons from the neighboring $\mathrm{Cr}$ atoms, leaving the third one free and so the n-type behavior.

We calculated therefore the DOS for defect free $\mathrm{CrN}$, CrN with a $\mathrm{Cr}$ vacancy, $\mathrm{CrN}$ with a $\mathrm{N}$ dumbbell, and with the most stable combined defect, and the results are shown in Fig. 3.6 around the Fermi level. Our calculations for the $\mathrm{N}$ dumbbell agree with the previous calculations by Mozafari et al. [86], where the valence band is shifted to lower energies compared to the defect free case. The Cr vacancy leads to a p-type semiconductor, with the valence band shifted to higher energies compared to the defect free DOS. For the combined defect, the situation is less clear, but there is a small shift towards higher energies, therefore we conclude that the behavior is p-type.

For a semiconductor, one should in principle investigate also charged defects; however, since in our calculations of defect free $\mathrm{CrN}$ the bandgap is small $(\sim 0.1$ $\mathrm{eV})$, the introduction of defects turns immediately the material into a metallic state. Moreover, experimentally it is not clear the magnitude of the gap [127], and defects are known to greatly influence its size. We therefore assume that, at this 


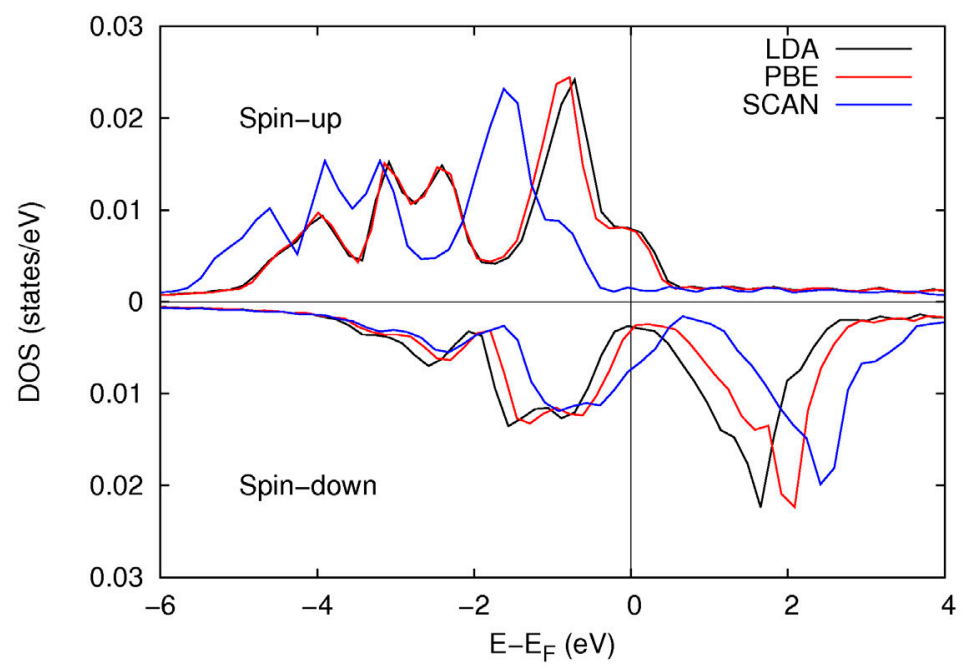

Figure 3.7: DOS for FM bcc Fe as calculated with LDA, PBE, and SCAN functional at the experimental lattice parameter. The DOS for spin-up electrons, which are the majority spin carriers, is represented in the top part of the plot, spin-down in the bottom. Figure adapted with permission from M. Ekholm, D. Gambino, H. J. M. Jönsson, F. Tasnádi, B. Alling, and I. A. Abrikosov, Physical Review B 98, 094413 (2018). Copyright 2018 by the American Physical Society.

concentrations, charged defects can be neglected.

\subsection{Assessing the SCAN functional for ferromag- netic elemental solids}

As discussed in Sec. 2.1, the main approximation in DFT is the exchange and correlation functional. The basic LDA and GGA-PBE have enabled the calculation of materials properties from first principles; however, they might not be accurate enough for more exotic systems, and there is a need of improving the accuracy of the calculations reducing always more the degree of approximation.

In the last few years, the SCAN functional [27] has received a lot of attention because it is in general able to improve the description of materials based on main group compounds [128]. However, it has been noticed as well that it has some issues with transition metal compounds (paper III, Ref. [128-130]).

In paper III, we compared results from LDA, PBE, and SCAN in the description of ground state properties of bcc $\mathrm{Fe}$, fcc $\mathrm{Ni}$, and hcp Co, all ferromagnetic metals.

In particular, for Fe, SCAN finds the right ground state as FM bcc Fe (as compared to LDA, which predicts NM fcc Fe to be lower in energy). It improves slightly equilibrium volume as compared to $\mathrm{PBE}$, however it predicts it to be a strong ferromagnet, with the majority-spin band fully occupied (see Fig. 3.7). 
The DOS in Fig. 3.7 are calculated at the experimental lattice parameter. The moments are best predicted by LDA at the experimental volume, although it severely underestimates the equilibrium volume.

For fcc Ni and hcp Co, SCAN does not give any kind of improvements as compared to PBE: it tends to underestimate the equilibrium volume and overestimate the magnetic moments. Also in these cases, SCAN shifts the majority-spin bands towards lower energies in the DOS.

Even though SCAN does not improve predictions as compared to PBE for elemental itinerant ferromagnets, it still provides an acceptable description of these systems. In Ref. [130], it has been shown that SCAN predicts Pd, which is a metal on the verge of ferromagnetism, to be a ferromagnet, whereas its ground state is nonmagnetic. This is a severe problem, and therefore magnetism should be a property to take more into account in the development of new exchange and correlation functionals.

\subsection{Interplay between vibrational, transverse, and longitudinal magnetic degrees of freedom in bcc Fe}

For a proper representation of reality, simulations that include all possible degrees of freedom (DOF) from first principles need to be carried out. For what concerns magnetic materials, many effects and their interplay need to be included, and methods that try to tackle this problem are usually tested on Fe because of its interesting physical properties.

As already mentioned, the description of magnetism is a challenge within DFT. Given its ground state focus, it is in general not straight-forward to investigate excited states as it can be the PM phase of a magnetic material. Nonetheless, integration of DFT with semiclassical models such as the DLM approach has lead to great advances in this field. The next step is to integrate this model within a larger framework that includes also vibrations. A further intrinsic complication is that the bare DLM model in DFT cannot properly account for longitudinal DOF, therefore thermodynamic models need to be added on top to describe this effect without resorting to more expensive higher-level theories.

In Fig. 3.8, the different DOF that can show a coupling in a magnetic system are schematized. In this framework, the effect of vibrations on the transversal magnetic degrees of freedom (arrow 1) have been discussed in the literature [63$65]$, showing the dependence of the exchange interactions on the distance between atoms and the effect of vibrations on the $J_{i j}$ s. The opposite influence (arrow 2) of finite temperature transversal magnetic DOF on vibrations has been shown to, e.g., induce a softening of the phonon modes in Fe [82], and to play a role on the shortening of phonon lifetimes in CrN [92]. The impact of LSF on the exchange interactions (arrow 3) has been investigated by Ruban et al. [68] in bcc Fe and fcc $\mathrm{Ni}$ in a DLM-CPA framework, as well as the behavior of LSF in a magnetically disordered background (arrow 4). 


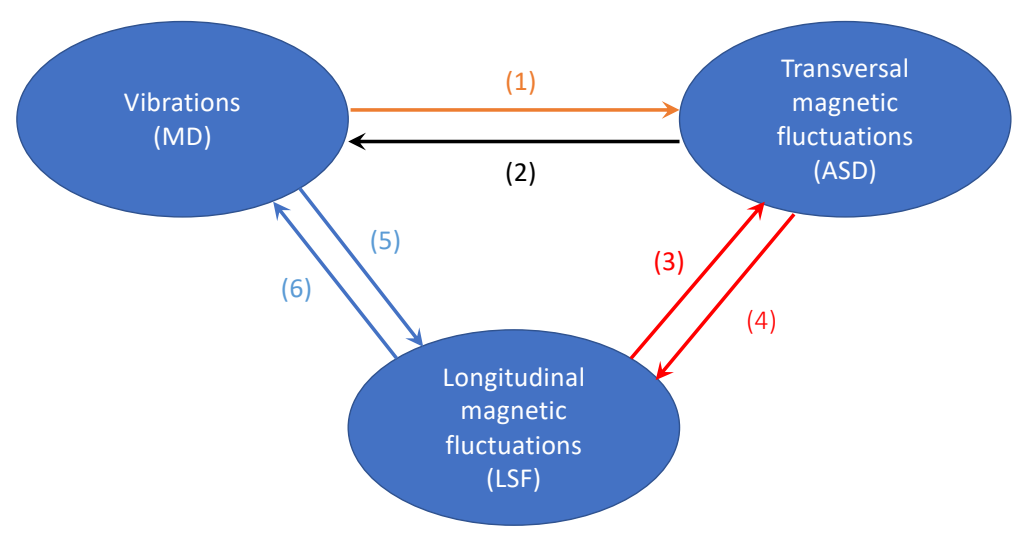

Figure 3.8: Schematic representation of the interplay between magnetic and vibrational degrees of freedom (DOF). Transversal and longitudinal magnetic DOF are here separated since complementary models need to be used to describe them, and they are expected to be on rather different timescales. The arrows indicate the influence of one type of DOF on the other. Arrow 1 (orange), 2 (black), and 3 and 4 (red) are referred to couplings that have been alredy discussed in literature in Ref. [63-65], [82, 92], and [68], respectively. Arrows 5 and 6, though, have not been discussed in literature. See text for a more detailed explanation of the different interplays.

Up to our knowledge, the interplay between vibrations and LSF has never been assessed carefully before. In Ref. [70], Dong et al. studied the effect of LSF on thermal expansion by means of a Debye-Grüneisen model. However, the LSF energy landscapes are derived in a DLM-CPA framework only accounting for volume expansion and no direct impact of the lattice vibrations. In Ref. [65], the exchange interactions for fcc $\mathrm{Ni}$ and fcc $\mathrm{Co}$ had been calculated with vibrational disorder, including also the LSF term of equation 2.33; however, the effect of vibrations on LSF was not discussed. In Ref. [87], DLM-MD simulations of bcc and fcc Fe were performed at different temperatures and it was shown that vibrations strongly affect the distribution of magnetic moments, however the influence of magnetic entropy on the size of the moments was not addressed.

To investigate this coupling, we have performed ASD-AIMD [92] simulations of bcc Fe in a $4 \times 4 \times 4$ supercell (128 atoms) at $\sim T_{C}=1043 \mathrm{~K}$, with distancedependent exchange interactions interpolated to the values reported in Ref. [65]. 


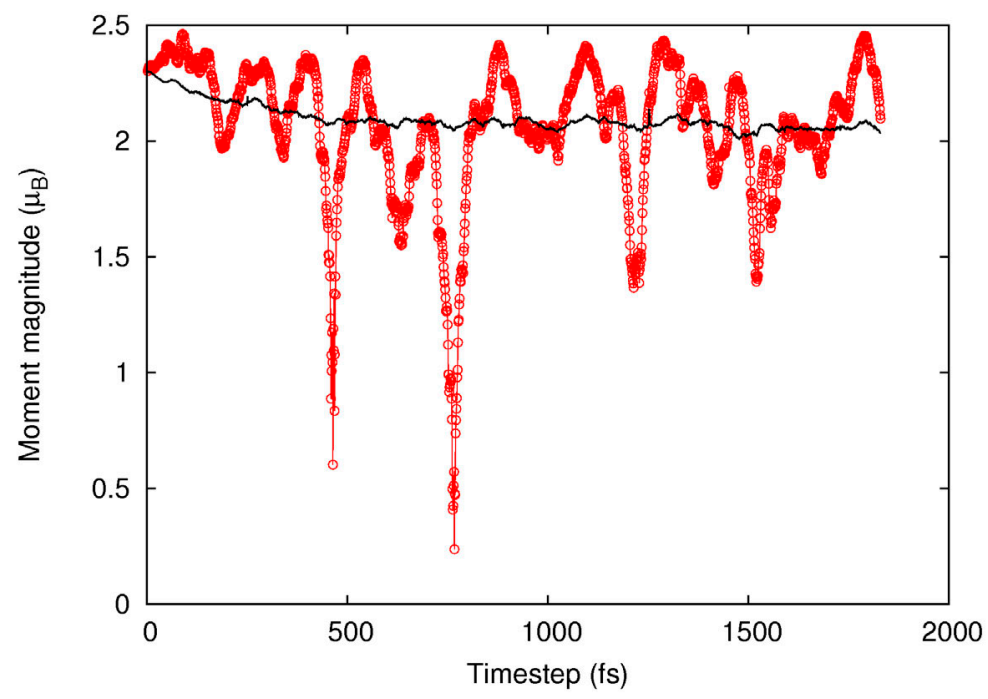

Figure 3.9: Average (black line) and a typical (red line and circles) moment size during an ASD-AIMD simulation for bcc Fe. The typical moment size drops to low values for a short time period, and then recovers to values around the average size.

We employed the Langevin thermostat with $\gamma=10 \mathrm{ps}^{-1}$ with a timestep of 1 fs for the AIMD part, whereas the ASD part, performed with the UppASD code $[66,131]$, is carried out with a timestep of 0.01 fs. In DFT calculations, we use an energy cutoff of $400 \mathrm{eV}$ for the expansion in plane-waves of the single-particle wavefunctions, and a $\Gamma$-centered $2 \times 2 \times 2$ k-points mesh. The moments are taken noncollinear and constrained with the method by Ma and Dudarev [28] in the directions given by the ASD code. We also employ a Fermi-Dirac smearing of the electronic wavefunctions corresponding to $T_{C}$. At this point, the moments' sizes can be considered as $0 \mathrm{~K}$ values, since the moments are constrained in direction but no thermal collective electronic excitation can be obtained directly with DFT.

After $\sim 500$ fs of thermalization, the average moment size seem to reach a stable value of $2.0-2.1 \mu_{B}$, as shown in Fig. 3.9 (black solid line). The development with time of a typical moment size is shown as well (red line with open circles), and it can be seen that it reduces quite drastically in two points of the simulation; however, it also manages to escape dynamically from this $\sim 0$ moment configuration.

In order to investigate how the vibrational disorder influences the LSF, we run a similar simulation with the same parameters but with a smaller supercell $(3 \times 3 \times 3$ conventional bcc cells, 54 atoms), and from this we take a configuration with a moment that goes close to zero in magnitude. From this fixed configuration of atoms and moments, we performed fixed-spin moment (FSM) calculations, i.e. in which also the size of the moments is constrained, and we change the moments' size one at a time. In this way, we can obtain the influence of both transverse magnetic and vibrational disorder on the LSF energy landscapes for each atom. 


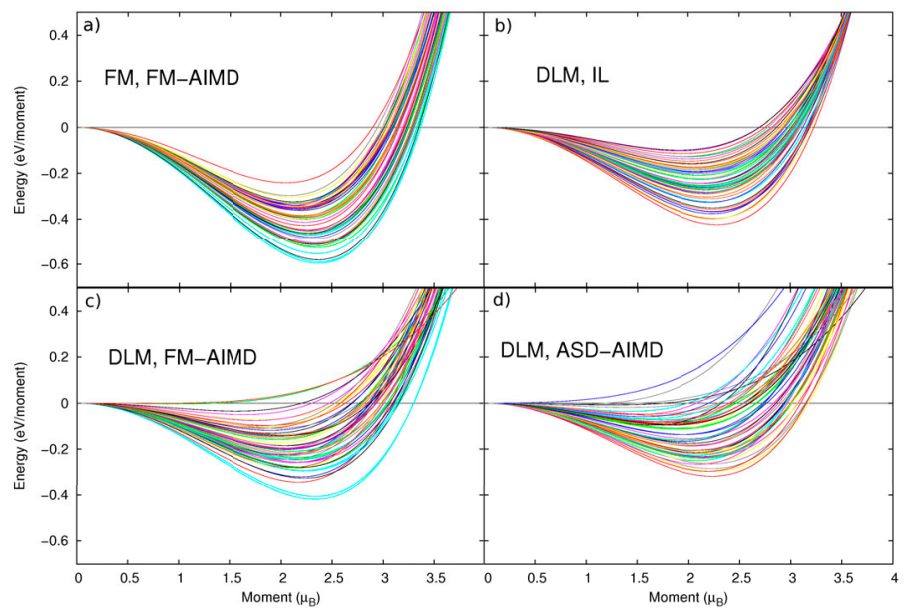

Figure 3.10: Landau interpolations of LSF energy landscapes obtained with FSM calculations for different magnetic states performed on different sets of atomic positions: a) FM state on FM-AIMD positions, b) DLM state on ideal lattice (IL) positions, c) DLM state on FM-AIMD positions, and d) DLM state on ASD-AIMD positions.

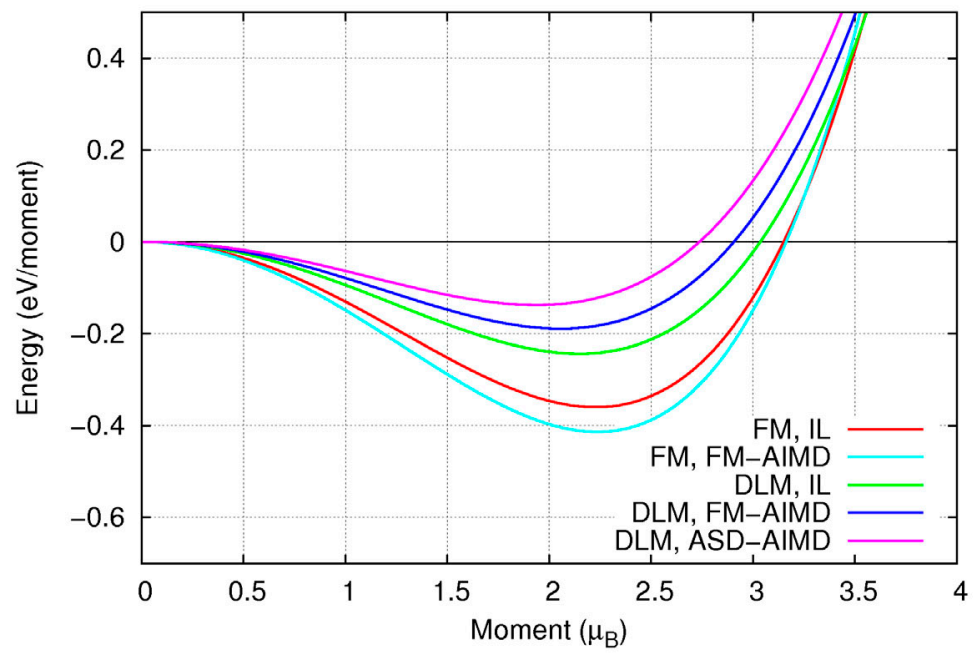

Figure 3.11: Landau interpolations of the average LSF landscapes calculated on atomic positions obtained from different means [ideal lattice positions (ILP), FMAIMD snapshot, and ASD-AIMD snapshot] and with different magnetic backgrounds (FM and DLM). 
We compare the same type of calculations using different set of positions (ideal lattice positions and an atomic configuration from FM-AIMD at the same temperature), as well as FM moments. The LSF energy landscape for each atom for FM moments on FM-AIMD positions, DLM moments on ideal lattice (IL) positions, DLM moments on FM-AIMD positions, and DLM moments on ASD-AIMD positions are shown in Fig. 3.10.

It is obvious that the energy landscapes calculated with DLM moments on ASD-AIMD positions are shallower than in all the other cases, with some moments that show a certain degree of itineracy (compare with Fig. 2.3), in contrast with the usual localized moment behavior of bcc Fe. Vibrations in general, both obtained from a FM-AIMD or ASD-AIMD simulation, induce very shallow landscapes on the DLM moments, as it can be seen comparing the two bottom figures in Fig. 3.10 with Fig.3.10b, which is calculated on ideal lattice position. At the same time, we can see that the FM magnetic background in combination with vibrations leads to deeper landscapes.

The average energy landscape for each of the previous cases are displayed in Fig. 3.11, where also the landscape for FM moments on ideal lattice positions is shown. Interestingly, vibrations make the minimum for FM moments deeper than on ideal lattice positions. As it could be expected, magnetic disorder make the minimum shallower than in FM state, and the addition of vibrations in FMAIMD and ASD-AIMD reduces it even further. Moreover, the minimum is moved towards smaller moments. These landscapes give rise at temperature $T \sim T_{C}$ to the probability distributions given in Fig. 3.12, which are calculated as the standard Boltzmann factor:

$$
P(m) \mathrm{d} m=\frac{m^{2} \exp \left(-\frac{E(m)}{k_{B} T}\right)}{Z} \mathrm{~d} m,
$$

calculated with $\mathrm{PSM}=m^{2}$. The probability distributions become broader and broader with increasing degree of disorder in the magnetic and vibrational DOF, as it could be inferred by the average landscapes; a decrease in the moment with maximum probability is also observed.

From the energy landscapes, one can numerically calculate thermodynamic properties such as average energy, entropy, free energy, and average moment as a function of temperature. In Fig. 3.13, we compare the results obtained from DLM on ideal lattice position (dashed lines and open symbols) and DLM on ASDAIMD position landscape (solid lines and full symbols), calculated with 3D PSM. In Fig. 3.13b, the average magnetic moment size is shown for the just mentioned positions (purple empty and cyan filled diamonds, respectively), as well as the average moment size calculated on ASD-AIMD positions calculated with 3D PSM and quadratic fitting of the landscape (filled blue squares), and with the 1D PSM with Landau fitting of the landscape (filled red circles).

The results given here are meaningful only for temperatures above $T_{C}$, since the LSF energy in DLM state employed here is motivated only in the high-temperature limit because of its single-site nature; values from $0 \mathrm{~K}$ are given only to make the trends more clear. We can see that the average energy is roughly the same for both landscapes, whereas there is a difference in entropic contribution at high 


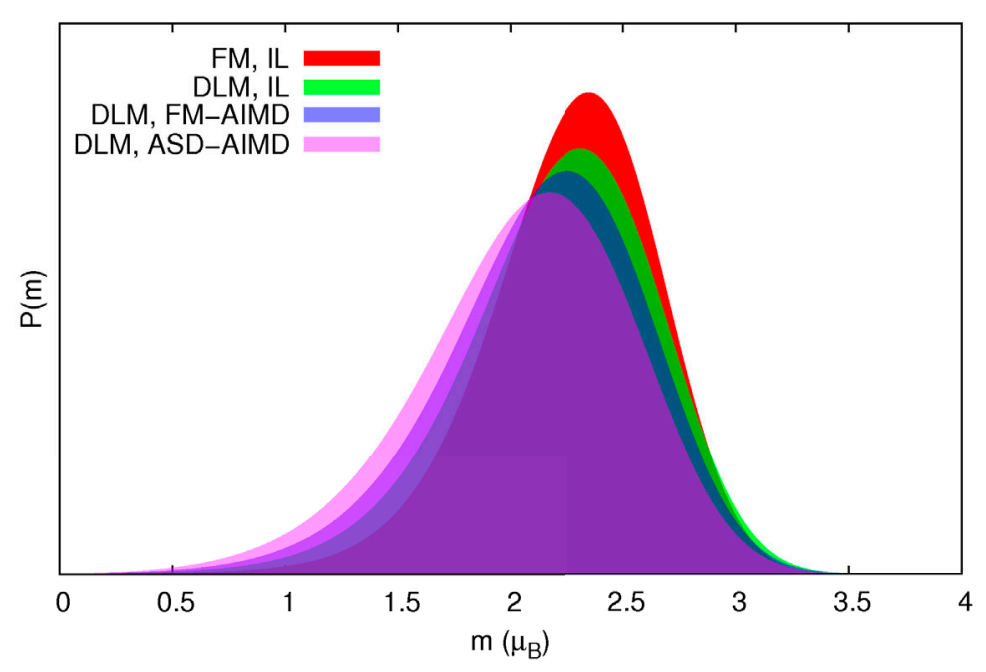

Figure 3.12: Probability distributions at $T^{\mathrm{mag}}=T_{C}^{\exp }$ obtained with Eq. 3.4 employing the average LSF energy landscapes of Fig. 3.11. Colors and labels are the same of Fig. 3.11.

temperature. This leads to a free energy difference of $\sim 10 \mathrm{meV} / \mathrm{atom}$, with the results calculated on ideal lattice positions at lower energies. It is also interesting to see the behavior of the average magnetic moment : for the ideal lattice, the value increases only slightly with temperature to $\sim 2.25 \mu_{B}$, whereas for the ASDAIMD positions the value increases more steeply going little beyond $\sim 2.1 \mu_{B}$. Although often an amount of $10 \mathrm{meV} /$ atom can be considered small, one needs to keep in mind that the largest Gibbs free energy difference between bcc and fcc Fe in the stability range of the fcc structure is experimentally estimated to be $1-3 \mathrm{meV} /$ atom [132], therefore it is crucial to assess these effects on the thermodynamics of Fe.

The choice of PSM dramatically changes the behavior of the moment magnitude: with the 1D PSM, the average moment decreases in size with temperature, going down to $\sim 1.6 \mu_{B}$, with a similar trend as shown in Ref. [68], although here the effect is stronger due to vibrations. A quadratic fitting of the energy landscape around the minimum, instead, leads to similar behavior of the Landau fitting, but this "harmonic" approximation makes the moments larger. It is not clear what should be the right trend for the moment size as a function of temperature (see, e.g., discussion in Ref. [87]), however the Landau fitting and 3D PSM are the most consistent, as previously discussed in Sec. 2.4.2. 


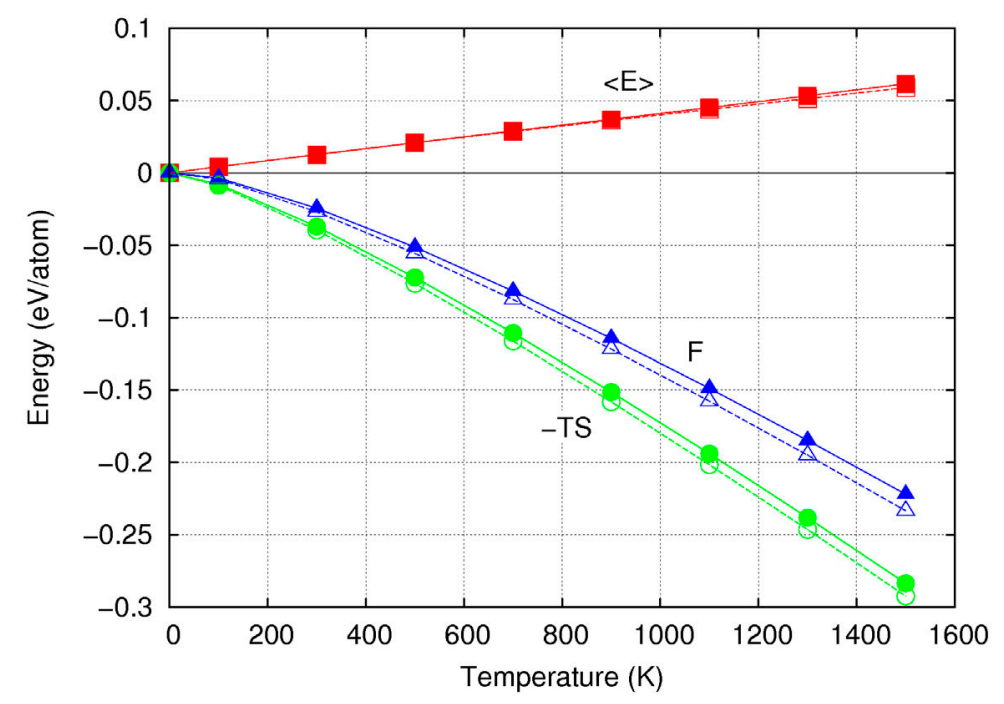

(a)

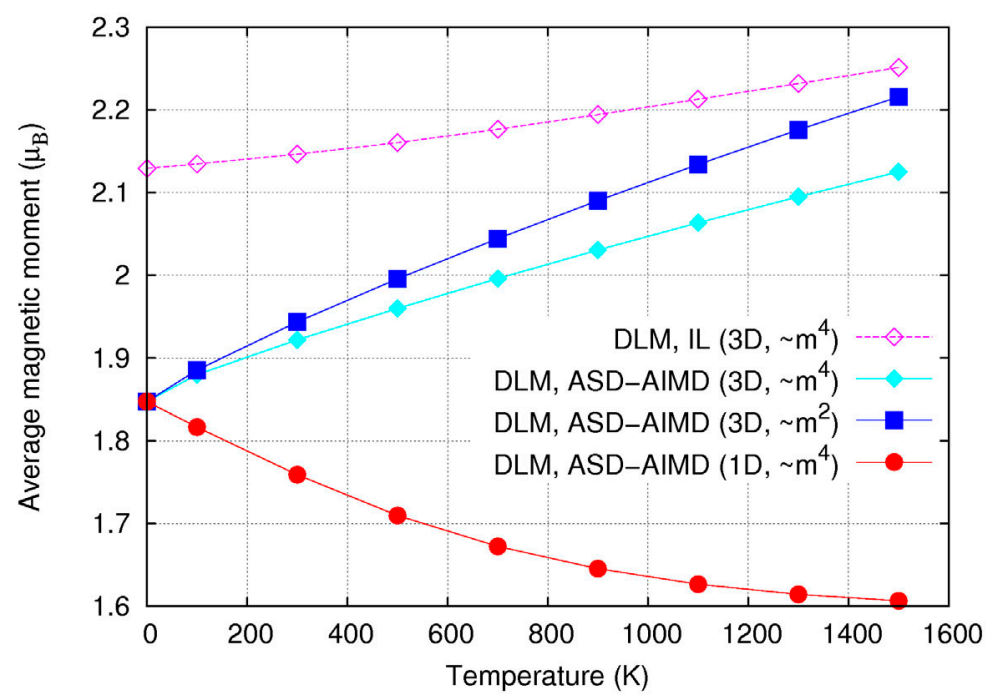

(b)

Figure 3.13: a) Average energy ( $<\mathrm{E}\rangle$, red), entropic contribution (-TS, green), and free energy $(\mathrm{F}$, blue) as a function of temperature for the average energy landscape calculated with DLM state on ideal lattice position (dashed lines and open symbols) and DLM state on ASD-AIMD positions (solid lines and full symbols) calculated with 3D PSM and Landau fitting. b) Average moment magnitude as a function of temperature calculated with 3D PSM and Landau fitting in DLM background on ideal lattice positions (purple empty diamonds) and on ASD-AIMD positions (cyan filled diamonds). The effect of different PSM is also shown taking into account the average moments calculated on ASD-AIMD positions with 3D PSM and a quadratic fitting of the landscape around the minimum (filled blue sqaures) and with 1D PSM and Landau fitting (filled red circles). 


\section{CHAPTER 4}

\section{Conclusions and outlooks}

In this work, structural and magnetic disorder in crystalline materials have been investigated by means of density functional theory and thermodynamic models. Methods to improve the description of these effects are developed and tested on relevant material systems.

In particular, disorder on the structural level is meant as presence of point defects in the otherwise perfect crystal lattice, or as substitutional random alloys. The diffusion rate of $\mathrm{Ti}$ vacancies in $\mathrm{TiN}$ at high temperature has been investigated by means of nonequilibrium ab initio molecular dynamics with the color diffusion algorithm. It is found that the jump rate follows an Arrhenius trend, and the energy barrier for diffusion decreases compared to $0 \mathrm{~K}$ calculations mainly because of thermal lattice expansion.

Point defects and random alloys are also studied in magnetic materials in the high temperature paramagnetic phase. We have developed a method based on the disordered local moments model to perform local lattice relaxations in the paramagnetic phase. It is found that relaxations in the relevant magnetic state affect the energetics of defects, leading to a reduction of vacancy formation energy in bcc $\mathrm{Fe}$ of $\sim 0.1 \mathrm{eV}$ and $\sim 0.2-0.4 \mathrm{eV}$ for $\mathrm{C}$ interstitial formation energy in octahedral and tetrahedral position, respectively, compared to relaxed positions in the ferromagnetic state. For substitutional random alloys, the impact of DLM relaxation is assessed in paramagnetic bcc $\mathrm{Fe}_{1-x} \mathrm{Cr}_{x}$ random alloys for compositions $x=0.25,0.5,0.75$, and a reduction in mixing enthalpy up to $0.05 \mathrm{eV} /$ atom $(\sim 10 \%)$ is found.

This relaxation method is also employed in the study of native point defects in paramagnetic $\mathrm{B} 1 \mathrm{CrN}$ that give overstoichiometry in $\mathrm{N}$. The most stable defect is found to be the Cr vacancy, that induces a p-type behavior in the DOS of the system. $\mathrm{N}$ interstitials in the form of $\mathrm{N}_{2}$ dumbbells are found to have a larger formation energy than Cr vacancy; however, these two types of defects 
show attractive interaction and lead to a weakly p-type semiconducting state. Since experimentally $\mathrm{CrN}$ is often synthesized in thin films with nonequilibrium growth conditions, we cannot rule out the possibility of having both defects in the system and their clustering. Further investigations are needed to clear out the main protagonist in $\mathrm{N}$ overstoichiometric $\mathrm{CrN}$.

The performances of the strongly constrained and appropriately normalized (SCAN) functional are then assessed on the ferromagnetic elemental solids bcc Fe, fcc Ni, and hep Co and compared with LDA and PBE results. It is found that SCAN slightly improve the description of bcc Fe for what concerns equilibrium volume and bulk modulus, but it worsen it for local magnetic moments, predicting bcc Fe to be a strong ferromagnet. For fcc Ni and hcp Co, SCAN worsen the results as compared to PBE. Further development of functionals for DFT that properly describe magnetism is therefore needed.

Finally, the interplay between magnetic and vibrational degrees of freedom in bcc Fe is investigated. A coupled atomistic spin dynamics-ab initio molecular dynamics simulation at the Curie temperature is carried out, and one snapshot is used then to assess the effect of lattice vibrations and transversal magnetic degrees of freedom on longitudinal spin fluctuations. It is found that both magnetic background and atomic positions affect the energy landscape of this degree of freedom, which is treated here in a mean-field fashion employing Landau expansion of the energy as a function of the moment size. The effect of different phase space measures and different fitting of the energy landscapes is also addressed. This study is an important preliminary step in the investigation by means of $a b$ initio techniques of phase stability of Fe at different temperatures with inclusion of all possible degrees of freedom, as well as in the establishment of methods to calculate free energies in magnetic materials in general. 


\section{Bibliography}

[1] P. Hohenberg and W. Kohn. "Inhomogeneous Electron Gas". In: Phys. Rev. 136 (3B 1964), B864-B871.

[2] W. Kohn and L. J. Sham. "Self-Consistent Equations Including Exchange and Correlation Effects". In: Phys. Rev. 140 (4A 1965), A1133-A1138.

[3] A. B. Mei et al. "Dynamic and structural stability of cubic vanadium nitride". In: Phys. Rev. B 91 (5 2015), p. 054101.

[4] Johan Klarbring and Sergei I. Simak. "Phase Stability of Dynamically Disordered Solids from First Principles". In: Phys. Rev. Lett. 121 (22 2018), p. 225702 .

[5] Björn Alling, Tobias Marten, and Igor A. Abrikosov. "Questionable collapse of the bulk modulus in CrN". In: Nature Materials 9 (Apr. 2010), 283 EP - .

[6] Neil W. Ashcroft and N. David Mermin. Solid state physics. Saunders College, 1976.

[7] A. Glensk et al. "Breakdown of the Arrhenius Law in Describing Vacancy Formation Energies: The Importance of Local Anharmonicity Revealed by Ab initio Thermodynamics". In: Phys. Rev. X 4 (1 2014), p. 011018.

[8] T. Diaz de la Rubia and M. W. Guinan. "New mechanism of defect production in metals: A molecular-dynamics study of interstitial-dislocation-loop formation in high-energy displacement cascades". In: Phys. Rev. Lett. 66 (21 1991), pp. 2766-2769.

[9] J Walker. "Optical absorption and luminescence in diamond". In: Reports on Progress in Physics 42.10 (1979), pp. 1605-1659.

[10] O. Barrera et al. "Understanding and mitigating hydrogen embrittlement of steels: a review of experimental, modelling and design progress from atomistic to continuum". In: Journal of Materials Science 53.9 (2018), pp. 62516290 . 
[11] M.A. Herman and H. Sitter. Molecular Beam Epitaxy: Fundamentals and Current Status. Springer Series in Materials Science. Springer Berlin Heidelberg, 1996.

[12] S. Frank et al. "Ni tracer diffusion in the B2-compound NiAl: influence of temperature and composition". In: Acta Materialia 49.8 (2001), pp. 1399 -1411 .

[13] B. Cantor et al. "Microstructural development in equiatomic multicomponent alloys". In: Materials Science and Engineering: A 375-377 (2004), pp. $213-218$.

[14] W. Lowrie. Fundamentals of Geophysics. Oct. 1997, p. 368.

[15] John B. Carlson. "Lodestone Compass: Chinese or Olmec Primacy?" In: Science 189.4205 (1975), pp. 753-760.

[16] Stephen Blundell. Magnetism in condensed matter. Oxford master series in condensed matter physics. Oxford Univ. Press, 2001.

[17] G. H. Wannier. "Antiferromagnetism. The Triangular Ising Net". In: Phys. Rev. 79 (2 1950), pp. 357-364.

[18] Jason S. Gardner, Michel J. P. Gingras, and John E. Greedan. "Magnetic pyrochlore oxides". In: Rev. Mod. Phys. 82 (1 2010), pp. 53-107.

[19] M. Pénicaud et al. "Calculated electronic band structure and magnetic moments of ferrites". In: Journal of Magnetism and Magnetic Materials 103.1 (1992), pp. $212-220$.

[20] P. A. M. Dirac. "Quantum mechanics of many electron systems". In: 123.792 (Apr. 1929), pp. 714-733.

[21] Richard M. Martin. Electronic Structure: Basic Theory and Practical Methods. Cambridge University Press, 2004.

[22] P. E. Blöchl. "Projector augmented-wave method". In: Phys. Rev. B 50 (24 1994), pp. 17953-17979.

[23] D. M. Ceperley and B. J. Alder. "Ground State of the Electron Gas by a Stochastic Method". In: Phys. Rev. Lett. 45 (7 1980), pp. 566-569.

[24] John P. Perdew, Kieron Burke, and Matthias Ernzerhof. "Generalized Gradient Approximation Made Simple". In: Phys. Rev. Lett. 77 (18 1996), pp. 3865-3868.

[25] John P. Perdew and Karla Schmidt. "Jacob's ladder of density functional approximations for the exchange-correlation energy". In: AIP Conference Proceedings 577.1 (2001), pp. 1-20. eprint: https://aip.scitation.org/ doi/pdf/10.1063/1.1390175.

[26] John A. Pople. "Quantum Chemical Models (Nobel Lecture)". In: Angewandte Chemie International Edition 38.13-14 (1999), pp. 1894-1902. eprint: https : / / onlinelibrary . wiley . com / doi / pdf / 10 . 1002 /\%28SICI \% 291521 - 3773\%2819990712\%2938\%3A13/14\%3C1894\%3A\%3AAID-ANIE1894\% 3E3. 0. CO\%3B2-H. 
[27] Jianwei Sun, Adrienn Ruzsinszky, and John P. Perdew. "Strongly Constrained and Appropriately Normed Semilocal Density Functional". In: Phys. Rev. Lett. 115 (3 2015), p. 036402.

[28] Pui-Wai Ma and S. L. Dudarev. "Constrained density functional for noncollinear magnetism". In: Phys. Rev. B 91 (5 2015), p. 054420.

[29] N. David Mermin. "Thermal Properties of the Inhomogeneous Electron Gas". In: Phys. Rev. 137 (5A 1965), A1441-A1443.

[30] Daan Frenkel and Berend Smit. Understanding molecular simulation. from algorithms to applications. Computational science series: 1. Academic Press, 2002.

[31] B. Grabowski et al. "Ab initio up to the melting point: Anharmonicity and vacancies in aluminum". In: Phys. Rev. B 79 (13 2009), p. 134106.

[32] Yilun Gong et al. "Temperature dependence of the Gibbs energy of vacancy formation of fcc Ni". In: Phys. Rev. B 97 (21 2018), p. 214106.

[33] Jörg Behler. "Perspective: Machine learning potentials for atomistic simulations". In: The Journal of Chemical Physics 145.17 (2016), p. 170901. eprint: https://doi.org/10.1063/1.4966192.

[34] D. G. Sangiovanni et al. "Efficient and accurate determination of latticevacancy diffusion coefficients via non equilibrium ab initio molecular dynamics". In: Phys. Rev. B 93 (9 2016), p. 094305.

[35] Philippe C. Aeberhard et al. "Ab initio Nonequilibrium Molecular Dynamics in the Solid Superionic Conductor $\mathrm{LiBH}_{4}$ ". In: Phys. Rev. Lett. 108 (9 2012), p. 095901.

[36] Wayne Nelson. Applied life data analysis. John Wiley \& Sons, Inc., New York, 1982.

[37] W. Kohn. "Density Functional and Density Matrix Method Scaling Linearly with the Number of Atoms". In: Phys. Rev. Lett. 76 (17 1996), pp. 31683171 .

[38] S. B. Zhang and John E. Northrup. "Chemical potential dependence of defect formation energies in GaAs: Application to Ga self-diffusion". In: Phys. Rev. Lett. 67 (17 1991), pp. 2339-2342.

[39] Chris G. Van de Walle et al. "First-principles calculations of solubilities and doping limits: Li, Na, and N in ZnSe". In: Phys. Rev. B 47 (15 1993), pp. $9425-9434$.

[40] Céline Varvenne et al. "Point defect modeling in materials: Coupling ab initio and elasticity approaches". In: Phys. Rev. B 88 (13 2013), p. 134102.

[41] Christoph Freysoldt, Jörg Neugebauer, and Chris G. Van de Walle. "Fully Ab Initio Finite-Size Corrections for Charged-Defect Supercell Calculations". In: Phys. Rev. Lett. 102 (1 2009), p. 016402. 
[42] Christoph Freysoldt, Jörg Neugebauer, and Chris G. Van de Walle. "Electrostatic interactions between charged defects in supercells". In: physica status solidi (b) 248.5 (2011), pp. 1067-1076. eprint: https: //onlinelibrary . wiley.com/doi/pdf/10.1002/pssb. 201046289.

[43] Henry Eyring. "The Activated Complex in Chemical Reactions". In: The Journal of Chemical Physics 3.2 (1935), pp. 107-115. eprint: https : / / doi.org/10.1063/1.1749604.

[44] Hannes Jónson, Greg Mills, and Karsten W. Jacobsen. "Nudged elastic band method for finding minimum energy paths of transitions". In: Classical and Quantum Dynamics in Condensed Phase Simulations, pp. 385404. eprint: https : / / www . worldscientific . com/doi / pdf / 10 . 1142 / 9789812839664_0016.

[45] Graeme Henkelman, Blas P. Uberuaga, and Hannes Jónsson. "A climbing image nudged elastic band method for finding saddle points and minimum energy paths". In: The Journal of Chemical Physics 113.22 (2000), pp. 99019904. eprint: https://doi.org/10.1063/1.1329672.

[46] Weinan E, Weiqing Ren, and Eric Vanden-Eijnden. "String method for the study of rare events". In: Phys. Rev. B 66 (5 2002), p. 052301.

[47] Weinan E, Weiqing Ren, and Eric Vanden-Eijnden. "Finite Temperature String Method for the Study of Rare Events". In: The Journal of Physical Chemistry B 109.14 (2005), pp. 6688-6693. eprint: https://doi.org/10. 1021/jp0455430.

[48] B. L. Gyorffy. "Coherent-Potential Approximation for a NonoverlappingMuffin-Tin-Potential Model of Random Substitutional Alloys". In: Phys. Rev. B 5 (6 1972), pp. 2382-2384.

[49] Alex Zunger et al. "Special quasirandom structures". In: Phys. Rev. Lett. 65 (3 1990), pp. 353-356.

[50] A V Ruban and I A Abrikosov. "Configurational thermodynamics of alloys from first principles: effective cluster interactions". In: Reports on Progress in Physics 71.4 (2008), p. 046501.

[51] J.M. Sanchez, F. Ducastelle, and D. Gratias. "Generalized cluster description of multicomponent systems". In: Physica A: Statistical Mechanics and its Applications 128.1 (1984), pp. $334-350$.

[52] A V Ruban and I A Abrikosov. "Configurational thermodynamics of alloys from first principles: effective cluster interactions". In: Reports on Progress in Physics 71.4 (2008), p. 046501.

[53] Walter Metzner and Dieter Vollhardt. "Correlated Lattice Fermions in $d=$ $\infty$ Dimensions". In: Phys. Rev. Lett. 62 (3 1989), pp. 324-327.

[54] Antoine Georges and Gabriel Kotliar. "Hubbard model in infinite dimensions". In: Phys. Rev. B 45 (12 1992), pp. 6479-6483.

[55] F. Aryasetiawan et al. "Frequency-dependent local interactions and lowenergy effective models from electronic structure calculations". In: Phys. Rev. B 70 (19 2004), p. 195104. 
[56] Takashi Miyake and F. Aryasetiawan. "Screened Coulomb interaction in the maximally localized Wannier basis". In: Phys. Rev. B 77 (8 2008), p. 085122.

[57] I.A. Abrikosov et al. "Recent progress in simulations of the paramagnetic state of magnetic materials". In: Current Opinion in Solid State and Materials Science 20.2 (2016), pp. $85-106$.

[58] A. I. Lichtenstein, M. I. Katsnelson, and G. Kotliar. "Finite-Temperature Magnetism of Transition Metals: An ab initio Dynamical Mean-Field Theory". In: Phys. Rev. Lett. 87 (6 2001), p. 067205.

[59] I. Leonov et al. "Electronic correlations determine the phase stability of iron up to the melting temperature". In: Scientific Reports 4 (2014), 5585 $\mathrm{EP}-$.

[60] Pascal Delange et al. "Large effects of subtle electronic correlations on the energetics of vacancies in $\alpha$-Fe". In: Phys. Rev. B 94 (10 2016), p. 100102.

[61] A.I. Liechtenstein et al. "Local spin density functional approach to the theory of exchange interactions in ferromagnetic metals and alloys". In: Journal of Magnetism and Magnetic Materials 67.1 (1987), pp. 65 -74.

[62] A. V. Ruban et al. "Atomic and magnetic configurational energetics by the generalized perturbation method". In: Phys. Rev. B 70 (12 2004), p. 125115.

[63] A. Lindmaa et al. "Exchange interactions in paramagnetic amorphous and disordered crystalline CrN-based systems". In: Phys. Rev. B 88 (5 2013), p. 054414 .

[64] Junqi Yin et al. "Effect of lattice vibrations on magnetic phase transition in bcc iron". In: Phys. Rev. B 86 (21 2012), p. 214423.

[65] A. V. Ruban and O. E. Peil. "Impact of thermal atomic displacements on the Curie temperature of $3 d$ transition metals". In: Phys. Rev. B 97 (17 2018), p. 174426.

[66] B Skubic et al. "A method for atomistic spin dynamics simulations: implementation and examples". In: Journal of Physics: Condensed Matter 20.31 (2008), p. 315203.

[67] L. M. Sandratskii. "Thermal magnetic properties of the Ni sublattice in halfmetallic NiMnSb: A theoretical study based on first-principles calculations". In: Phys. Rev. B 78 (9 2008), p. 094425.

[68] A. V. Ruban et al. "Temperature-induced longitudinal spin fluctuations in Fe and Ni". In: Phys. Rev. B 75 (5 2007), p. 054402.

[69] A. V. Ruban, A. B. Belonoshko, and N. V. Skorodumova. "Impact of magnetism on Fe under Earth's core conditions". In: Phys. Rev. B 87 (1 2013), p. 014405.

[70] Zhihua Dong et al. "Longitudinal spin fluctuation contribution to thermal lattice expansion of paramagnetic Fe". In: Phys. Rev. B 95 (5 2017), p. 054426 . 
[71] Sergii Khmelevskyi. "Longitudinal integration measure in classical spin space and its application to first-principle based simulations of ferromagnetic metals". In: Journal of Magnetism and Magnetic Materials 461 (2018), pp. $14-18$.

[72] N. M. Rosengaard and Börje Johansson. "Finite-temperature study of itinerant ferromagnetism in Fe, Co, and Ni". In: Phys. Rev. B 55 (22 1997), pp. $14975-14986$.

[73] A. L. Wysocki, J. K. Glasbrenner, and K. D. Belashchenko. "Thermodynamics of itinerant magnets in a classical spin-fluctuation model". In: Phys. Rev. B 78 (18 2008), p. 184419.

[74] Fan Pan et al. "Extended spin model in atomistic simulations of alloys". In: Phys. Rev. B 95 (18 2017), p. 184432.

[75] J. Hubbard. "The magnetism of iron". In: Phys. Rev. B 19 (5 1979), pp. 26262636.

[76] J. Hubbard. "Magnetism of iron. II". In: Phys. Rev. B 20 (11 1979), pp. 45844595.

[77] J. Hubbard. "Magnetism of nickel". In: Phys. Rev. B 23 (11 1981), pp. 59745977.

[78] Hideo Hasegawa. "Single-Site Functional-Integral Approach to ItinerantElectron Ferromagnetism". In: Journal of the Physical Society of Japan 46.5 (1979), pp. 1504-1514. eprint: https://doi .org/10.1143/JPSJ . 46. 1504.

[79] Hideo Hasegawa. "Single-Siet Spin Fluctuation Theory of Itinerant-Electron Systems with Narrow Bands". In: Journal of the Physical Society of Japan 49.1 (1980), pp. 178-188. eprint: https : //doi .org/10.1143/JPSJ . 49 . 178.

[80] B L Gyorffy et al. "A first-principles theory of ferromagnetic phase transitions in metals". In: Journal of Physics F: Metal Physics 15.6 (1985), p. 1337.

[81] B. Alling, T. Marten, and I. A. Abrikosov. "Effect of magnetic disorder and strong electron correlations on the thermodynamics of CrN". In: Phys. Rev. B 82 (18 2010), p. 184430.

[82] F. Körmann et al. "Atomic forces at finite magnetic temperatures: Phonons in paramagnetic iron". In: Phys. Rev. B 85 (12 2012), p. 125104.

[83] B. Alling et al. "Strong electron correlations stabilize paramagnetic cubic $\mathrm{Cr}_{1-x} \mathrm{Al}_{x} \mathrm{~N}$ solid solutions". In: Applied Physics Letters 102.3 (2013), p. 031910. eprint: https://doi.org/10.1063/1.4788747.

[84] Peter Steneteg, Björn Alling, and Igor A. Abrikosov. "Equation of state of paramagnetic CrN from ab initio molecular dynamics". In: Phys. Rev. B 85 (14 2012), p. 144404.

[85] Nina Shulumba et al. "Vibrational free energy and phase stability of paramagnetic and antiferromagnetic CrN from ab initio molecular dynamics". In: Phys. Rev. B 89 (17 2014), p. 174108. 
[86] E. Mozafari et al. "Role of $\mathrm{N}$ defects in paramagnetic $\mathrm{CrN}$ at finite temperatures from first principles". In: Phys. Rev. B 91 (9 2015), p. 094101.

[87] B. Alling et al. "Strong impact of lattice vibrations on electronic and magnetic properties of paramagnetic Fe revealed by disordered local moments molecular dynamics". In: Phys. Rev. B 93 (22 2016), p. 224411.

[88] N. Sandberg et al. "Modeling of the magnetic free energy of self-diffusion in bcc Fe". In: Phys. Rev. B 92 (18 2015), p. 184102.

[89] A. V. Ponomareva, Yu. N. Gornostyrev, and I. A. Abrikosov. "Ab initio calculation of the solution enthalpies of substitutional and interstitial impurities in paramagnetic fcc Fe". In: Phys. Rev. B 90 (1 2014), p. 014439.

[90] A. V. Ruban and V. I. Razumovskiy. "Spin-wave method for the total energy of paramagnetic state". In: Phys. Rev. B 85 (17 2012), p. 174407.

[91] Hong Ding, Vsevolod I. Razumovskiy, and Mark Asta. "Self diffusion anomaly in ferromagnetic metals: A density-functional-theory investigation of magnetically ordered and disordered Fe and Co". In: Acta Materialia 70 (2014), pp. $130-136$.

[92] Irina Stockem et al. "Anomalous Phonon Lifetime Shortening in Paramagnetic CrN Caused by Spin-Lattice Coupling: A Combined Spin and Ab Initio Molecular Dynamics Study". In: Phys. Rev. Lett. 121 (12 2018), p. 125902 .

[93] G. Kresse and J. Furthmüller. "Efficient iterative schemes for ab initio totalenergy calculations using a plane-wave basis set". In: Phys. Rev. B 54 (16 1996), pp. 11169-11186.

[94] G. Kresse and J. Furthmüller. "Efficient iterative schemes for ab initio totalenergy calculations using a plane-wave basis set". In: Phys. Rev. B 54 (16 1996), pp. 11169-11186.

[95] G. Kresse and D. Joubert. "From ultrasoft pseudopotentials to the projector augmented-wave method". In: Phys. Rev. B 59 (3 1999), pp. 1758-1775.

[96] R. Armiento and A. E. Mattsson. "Functional designed to include surface effects in self-consistent density functional theory". In: Phys. Rev. B 72 (8 2005), p. 085108.

[97] S. L. Dudarev et al. "Electron-energy-loss spectra and the structural stability of nickel oxide: An LSDA+U study". In: Phys. Rev. B 57 (3 1998), pp. $1505-1509$.

[98] L. Ning, S.C. Veldhuis, and K. Yamamoto. "Investigation of wear behavior and chip formation for cutting tools with nano-multilayered $\mathrm{TiAlCrN} / \mathrm{NbN}$ PVD coating". In: International Journal of Machine Tools and Manufacture 48.6 (2008), pp. $656-665$.

[99] Jian-Long Ruan et al. "Investigation of substrate bias effects on the reactively sputtered ZrN diffusion barrier films". In: Ceramics International 35.5 (2009), pp. $1999-2005$. 
[100] Paul H. Mayrhofer et al. "Microstructural design of hard coatings". In: Progress in Materials Science 51.8 (2006), pp. 1032 -1114.

[101] H. Kindlund et al. "Toughness enhancement in hard ceramic thin films by alloy design". In: APL Materials 1.4 (2013), p. 042104. eprint: https : //doi.org/10.1063/1.4822440.

[102] V.V. Uglov et al. "Structural and mechanical stability upon annealing of arc-deposited Ti-Zr-N coatings". In: Surface and Coatings Technology 202.11 (2008), pp. $2394-2398$.

[103] G. Abadias et al. "Reactive magnetron cosputtering of hard and conductive ternary nitride thin films: Ti-Zr-N and Ti-Ta-N". In: Journal of Vacuum Science \& Technology A 28.4 (2010), pp. 541-551. eprint: https ://doi . org/10.1116/1.3426296.

[104] L Hultman. "Thermal stability of nitride thin films". In: Vacuum 57.1 (2000), pp. $1-30$.

[105] Carina Höglund et al. "Effects of volume mismatch and electronic structure on the decomposition of ScAlN and TiAlN solid solutions". In: Phys. Rev. B 81 (22 2010), p. 224101.

[106] Paul H. Mayrhofer et al. "Self-organized nanostructures in the Ti-Al-N system". In: Applied Physics Letters 83.10 (2003), pp. 2049-2051. eprint: https://doi.org/10.1063/1.1608464.

[107] L.E. Toth. Transition metal carbides and nitrides. Refractory materials. Academic Press, 1971.

[108] J. E. Sundgren et al. " $\mathrm{TiN}_{\mathrm{x}}(0.6<\mathrm{x}<1.2)$ : Atomic arrangements, Electronic structure and recent results on crystal growth and physical properties of epitaxial layers". In: AIP Conference Proceedings 149.1 (1986), pp. 95-115. eprint: https://aip.scitation.org/doi/pdf/10.1063/1.36035.

[109] L. Tsetseris et al. "Structure and interaction of point defects in transitionmetal nitrides". In: Phys. Rev. B 76 (22 2007), p. 224107.

[110] "Chapter 10 - A Sample Completion Technique for Censored Samples". In: Order Statistics and Inference. Ed. by N. Balakrishnan and A. Clifford Cohen. San Diego: Academic Press, 1991, pp. 329 -340.

[111] D. G. Sangiovanni et al. "Nitrogen vacancy, self-interstitial diffusion, and Frenkel-pair formation/dissociation in B1 TiN studied by ab initio and classical molecular dynamics with optimized potentials". In: Phys. Rev. B 91 (5 2015), p. 054301.

[112] L. Hultman, C. Engström, and M. Odén. "Mechanical and thermal stability of TiN/NbN superlattice thin films". In: Surface and Coatings Technology 133-134 (2000), pp. $227-233$.

[113] H.-E. Schaefer et al. "Vacancy formation in iron investigated by positron annihilation in thermal equilibrium". In: Scripta Metallurgica 11.9 (1977), pp. $803-809$. 
[114] S M Kim and W J L Buyers. "Vacancy formation energy in iron by positron annihilation". In: Journal of Physics F: Metal Physics 8.5 (1978), p. L103.

[115] H. Matter, J. Winter, and W. Triftshäuser. "Phase transformations and vacancy formation energies of transition metals by positron annihilation". In: Applied physics 20.2 (1979), pp. 135-140.

[116] L. De Schepper et al. "Positron annihilation on pure and carbon-doped $\alpha$ iron in thermal equilibrium". In: Phys. Rev. B 27 (9 1983), pp. 5257-5269.

[117] H.-E. Schaefer. "Investigation of Thermal Equilibrium Vacancies in Metals by Positron Annihilation". In: physica status solidi (a) 102.1 (1987), pp. 4765. eprint: https://onlinelibrary.wiley.com/doi/pdf/10.1002/pssa. 2211020104.

[118] Nicolas Mounet and Nicola Marzari. "First-principles determination of the structural, vibrational and thermodynamic properties of diamond, graphite, and derivatives". In: Phys. Rev. B 71 (20 2005), p. 205214.

[119] Fabien Tran, Julia Stelzl, and Peter Blaha. "Rungs 1 to 4 of DFT Jacob's ladder: Extensive test on the lattice constant, bulk modulus, and cohesive energy of solids". In: The Journal of Chemical Physics 144.20 (2016), p. 204120. eprint: https://doi.org/10.1063/1.4948636.

[120] P. Olsson et al. "Ab initio formation energies of Fe-Cr alloys". In: Journal of Nuclear Materials 321.1 (2003), pp. $84-90$.

[121] P. Olsson, I. A. Abrikosov, and J. Wallenius. "Electronic origin of the anomalous stability of Fe-rich bcc Fe-Cr alloys". In: Phys. Rev. B 73 (10 2006), p. 104416.

[122] Costel Constantin et al. "Metal/semiconductor phase transition in chromium nitride(001) grown by rf-plasma-assisted molecular-beam epitaxy". In: Applied Physics Letters 85.26 (2004), pp. 6371-6373. eprint: https : //doi . org/10.1063/1.1836878.

[123] A. Ney et al. "Magnetic properties of epitaxial CrN films". In: Applied Physics Letters 89.11 (2006), p. 112504. eprint: https : //doi .org/10 . 1063/1.2352795.

[124] Francisco Rivadulla et al. "Reduction of the bulk modulus at high pressure in CrN". In: Nature Materials 8 (Oct. 2009), 947 EP -.

[125] Koichi Momma and Fujio Izumi. "VESTA3 for three-dimensional visualization of crystal, volumetric and morphology data". In: Journal of Applied Crystallography 44.6 (2011), pp. 1272-1276.

[126] Arnaud le Febvrier et al. "P-type Al-doped Cr-deficient CrN thin films for thermoelectrics". In: Applied Physics Express 11.5 (2018), p. 051003.

[127] Aditi Herwadkar and Walter R. L. Lambrecht. "Electronic structure of CrN: A borderline Mott insulator". In: Phys. Rev. B 79 (3 2009), p. 035125.

[128] Yubo Zhang et al. "Efficient first-principles prediction of solid stability: Towards chemical accuracy". In: npj Computational Materials 4.1 (2018), p. 9 . 
[129] Aldo H. Romero and Matthieu J. Verstraete. "From one to three, exploring the rungs of Jacob's ladder in magnetic alloys". In: The European Physical Journal B 91.8 (2018), p. 193.

[130] Yuhao Fu and David J. Singh. "Applicability of the Strongly Constrained and Appropriately Normed Density Functional to Transition-Metal Magnetism". In: Phys. Rev. Lett. 121 (20 2018), p. 207201.

[131] Olle Eriksson et al. Atomistic Spin Dynamics: Foundations and Applications. Oxford: Oxford University Press, 2017, p. 272.

[132] Qing Chen and Bo Sundman. "Modeling of thermodynamic properties for Bcc, Fcc, liquid, and amorphous iron". In: Journal of Phase Equilibria 22.6 (2001), pp. 631-644. 
List of included publications

[I] Nonequilibrium ab initio molecular dynamics determination of Ti monovacancy migration rates in $\mathrm{B} 1 \mathrm{TiN}$.

D. Gambino, D. G. Sangiovanni, B. Alling, and I. A. Abrikosov.

Physical Review B, 96, 104306 (2017).

[II] Lattice relaxations in disordered Fe-based materials in the paramagnetic state from first principles.

D. Gambino and B. Alling.

Physical Review B, 98, 064105 (2018).

[III] Assessing the SCAN functional for itinerant electron ferromagnets.

M. Ekholm, D. Gambino, H. J. M. Jönsson, F. Tasnádi, B. Alling, and I. A. Abrikosov.

Physical Review B, 98, 094413 (2018). 
Not included publications

Effect of dispersion corrections on ab initio predictions of graphite and diamond properties under pressure.

I. Mosyagin, D. Gambino, D. G. Sangiovanni, I. A. Abrikosov, and N. M. Caffrey. Physical Review B, 98, 174103 (2018). 


\section{CHAPTER 5}

\section{Summary of included publications}

\section{Copyrights}

Copyrights of papers I, II, and III belong to the American Physical Society.

\section{Paper I}

Nonequilibrium ab initio molecular dynamics determination of Ti monovacancy migration rates in $\mathrm{B} 1 \mathrm{TiN}$.

\section{Summary}

In this paper, the diffusion rate of $\mathrm{Ti}$ vacancies in $\mathrm{TiN}$ is studied by means of nonequilibrium $a b$ initio molecular dynamics with the color diffusion algorithm extended to the exponential regime. The analysis of jump occurrence time is performed with exponential and gamma distributions, so that a well defined uncertainty on the jump rates is derived, very important for stochastic processes such as vacancy-mediated diffusion. The behavior as a function of temperature follows an Arrhenius trend.

\section{Author's contribution}

I took part in planning of the project, performed all the calculations, participated in the analysis of the results, and was responsible for writing the manuscript.

\section{Paper II}

Lattice relaxations in disordered Fe-based materials in the paramagnetic state from first principles. 


\section{Summary}

A method to perform lattice relaxations in magnetic materials in the high temperature paramagnetic state is developed, based on the disordered local moments approach. The method is tested on Fe-based materials, in particular the formation energy of monovacancies and $\mathrm{C}$ interstitials in octahedral position in bcc Fe, as well as the mixing enthalpy of bcc $\mathrm{Fe}_{1-x} \mathrm{Cr}_{x}$ random alloys for compositions $x=0.25,0.50$, and 0.75 . Relaxations in the DLM state are proven to give non negligible contributions to the energetics of these systems.

\section{Author's contribution}

I took part in planning of the project, implemented the code for performing relaxation, performed all the calculations, participated in the analysis of the results, and was responsible for writing the manuscript.

\section{Paper III}

Assessing the SCAN functional for itinerant electron ferromagnets.

\section{Summary}

The strongly constrained appropriately normalized (SCAN) functional is tested on the ferromagnetic elemental solids bcc $\mathrm{Fe}$, fcc $\mathrm{Ni}$, and hcp Co, and compared with the LDA and PBE functionals. It is found that SCAN slightly improves the description of equilibrium volume and bulk modulus of bcc Fe as compared to PBE, but it predicts it to be a strong ferromagnet and overestimates the magnetic moments. For fcc Ni and hcp Co, SCAN performs worse than PBE also on equilibrium volume and bulk modulus, besides a consistent overestimation of the magnetic moments.

\section{Author's contribution}

I did all the VASP calculations, and took part in the analysis of the results. 


\section{Papers}

The papers associated with this thesis have been removed for copyright reasons. For more details about these see:

http://urn.kb.se/resolve?urn=urn:nbn:se:liu:diva-156835 


\section{FACULTY OF SCIENCE AND ENGINEERING}

Linköping Studies in Science and Technology, Licentiate Thesis No. 1837, 2019 Department of Physics, Chemistry and Biology (IFM)

Linköping University

SE-58183 Linköping, Sweden

www.liu.se 